\title{
Model reference control using sliding mode with Hamiltonian dynamics
}

\author{
R. J. Stonier J. Zajaczkowski*
}

(Received 11 December 2000; revised 24 February 2003)

\begin{abstract}
Model reference control and model reference adaptive control has since its inception, found application in a wide range of applications from the control of simple mechanical structures to the more complex robotic manipulators. Sliding mode techniques largely simplify the task of tracking the reference model and are capable of accommodating the uncertainties present in the dynamics of the system. In this paper we are concerned with model tracking in finite time for plant and reference model which are given in Hamiltonian format. The method is applied to nonlinear plant and linear model, with particular application to robot control. We also include the addition of a stabilising supervisory controller in terms of the Hamiltonian of the reference model.
\end{abstract}

*School of Mathematics and Decision Science, Central Queensland University, Rockhampton, Queensland 4702, AUsTRALIA. mailto:r.stonier@cqu.edu.au

See http://anziamj.austms.org.au/V45/E056/home.html for this article, (C) Austral. Mathematical Soc. 2003. Published December 14, 2003 ISSN 1446-8735 


\section{Contents}

1 Introduction

E2

2 Model reference control MRC E5

2.1 Assumptions . . . . . . . . . . . . . . . E E

2.2 Control law $-\mathrm{MRC} \ldots \ldots \ldots \ldots$ E6

2.3 Robot illustration $-\mathrm{MRC} \ldots \ldots \ldots$ E8

2.4 Control law for robotic example $-\mathrm{MRC} \ldots \ldots$ E12

3 Model reference adaptive control - MRAC $\quad$ E16

3.1 Control and adaptation law in MRAC . . . . . . . . E E17

3.2 Convergence . . . . . . . . . . . . . . . . . E18

3.3 Robot illustration - MRAC . . . . . . . . . . . . . E19

3.4 Control and adaptation law . . . . . . . . . E20

4 Stability $\quad$ E24

4.1 Supervisory controller . . . . . . . . . . . . E24

4.2 Stable controller for sliding mode . . . . . . . . E26

4.3 Robot illustration . . . . . . . . . . . . . . E31

4.4 Computer simulation with supervisory controller . . . . . E35

4.5 Asymptotic stability versus sliding mode . . . . . . . . E35

5 Conclusions

E39

References

E40

\section{Introduction}

Model reference control (MRC) and model reference adaptive control (MRAC) has a wide range of applications from the control of simple mechanical structures to the control of complex robotic manipulators. Specifically, it has 
proven its practicality in control systems with constant or slowly-varying parameters. A whole range of different formalisms may be used to synthesise an adaptation mechanism. Sliding mode techniques largely simplify the task of tracking the reference model and are capable of accommodating the uncertainties present in the dynamics of the system. We use the Lyapunov method and sliding mode dynamics to determine controllers for (adaptive) tracking in finite time and also give sufficient conditions to ensure that the resulting system is stable.

Using the Hamiltonian formulation immediately provides a control system in standard state space format. Further, position and momentum coordinates are conjugate variables and the Hamiltonian itself is related to the energy of the system (in conservative systems it represents directly total energy of the system). Model reference control in Hamiltonian formulation [3] uses a Hamiltonian function and Lyapunov asymptotic stability techniques for MRC with no reference to sliding mode. (An earlier formulation has been presented by Skowronski [2].)

We begin by setting the Hamiltonian structure for the plant and model. The plant is described by Hamiltonian canonical equations:

$$
\begin{aligned}
\dot{q}_{i} & =\frac{\partial H_{p}(\boldsymbol{q}, \boldsymbol{p}, \boldsymbol{a})}{\partial p_{i}}, \\
\dot{p}_{i} & =-\frac{\partial H_{p}(\boldsymbol{q}, \boldsymbol{p}, \boldsymbol{a})}{\partial q_{i}}+Q_{i}^{D}(\boldsymbol{q}, \boldsymbol{p}, \boldsymbol{d})+Q_{i}^{F}(\boldsymbol{q}, \boldsymbol{p}, \boldsymbol{a}, \boldsymbol{u}),
\end{aligned}
$$

where $i=1, \ldots, n$ (we assume that $n=2$ ) and $H_{p}$ is the Hamiltonian of the system, $Q_{i}^{D}$ is a damping force, $Q_{i}^{F}$ is an external force, $\boldsymbol{u}$ is a control vector, and $\boldsymbol{a}=\left[a_{1}, \ldots, a_{k}\right], k \leq n$, is a vector of adjustable plant parameters. For technical reason we expand the vector $\boldsymbol{a}$ to a dimension $n$ by assuming $a_{j}=0$ for $n \geq j>k$. We also assume that damping coefficients represented by vector $\boldsymbol{d}=\left[d_{1}, \ldots, d_{n}\right]$ may be polluted by uncertainty, and thus not known in exact form. The output state is $\boldsymbol{x}=\left[\begin{array}{ll}\boldsymbol{q} & \boldsymbol{p}\end{array}\right]^{T}$. In MRC we assume no uncertainty present in the system, and no adaptation parameters. 
The reference model is designed as another Hamiltonian system with Hamiltonian $H_{m}$, and output $\boldsymbol{x}_{m}=\left[\begin{array}{ll}\boldsymbol{q}_{m} & \boldsymbol{p}_{m}\end{array}\right]^{T}$. In this model the damping force and external force are assumed to be known functions of time and are such that the reference model is Lagrange stable, that is, the model output is bounded:

$$
\begin{aligned}
\dot{q}_{m_{i}} & =\frac{\partial H_{m}\left(\boldsymbol{q}_{m}, \boldsymbol{p}_{m}\right)}{\partial p_{m_{i}}} \\
\dot{p}_{m_{i}} & =-\frac{\partial H_{m}\left(\boldsymbol{q}_{m}, \boldsymbol{p}_{m}\right)}{\partial q_{m_{i}}}+Q_{m_{i}}^{D}\left(\boldsymbol{q}_{m}, \boldsymbol{p}_{m}, \boldsymbol{d}_{m}\right)+Q_{m_{i}}^{F}\left(\boldsymbol{q}_{m}, \boldsymbol{p}_{m}, \boldsymbol{u}_{m}\right) .
\end{aligned}
$$

Define the state error vector $\boldsymbol{e}=\left[\begin{array}{ll}\boldsymbol{e}_{q} & \boldsymbol{e}_{p}\end{array}\right]^{T}$ as

$$
\begin{aligned}
e_{q_{i}}(t) & =q_{i}(t)-q_{m_{i}}(t), \\
e_{p_{i}}(t) & =p_{i}(t)-p_{m_{i}}(t), \quad i=1, \ldots, n .
\end{aligned}
$$

The rate of change of $\boldsymbol{e}$ is

$$
\begin{aligned}
\dot{\boldsymbol{e}}_{q_{i}}= & \frac{\partial H_{p}(\boldsymbol{q}, \boldsymbol{p}, \boldsymbol{a})}{\partial p_{i}}-\frac{\partial H_{m}\left(\boldsymbol{q}_{m}, \boldsymbol{p}_{m}\right)}{\partial p_{m_{i}}}, \\
\dot{\boldsymbol{e}}_{p_{i}}= & -\frac{\partial H_{p}(\boldsymbol{q}, \boldsymbol{p}, \boldsymbol{a})}{\partial q_{i}}+\frac{\partial H_{m}\left(\boldsymbol{q}_{m}, \boldsymbol{p}_{m}\right)}{\partial q_{m_{i}}}+Q_{i}^{D}(\boldsymbol{q}, \boldsymbol{p}, \boldsymbol{d}) \\
& -Q_{m_{i}}^{D}\left(\boldsymbol{q}_{m}, \boldsymbol{p}_{m}, \boldsymbol{d}_{m}\right)+Q_{i}^{F}(\boldsymbol{q}, \boldsymbol{p}, \boldsymbol{a}, \boldsymbol{u})-Q_{m_{i}}^{F}\left(\boldsymbol{q}_{m}, \boldsymbol{p}_{m}, \boldsymbol{u}_{m}\right),
\end{aligned}
$$

$i=1, \ldots, n$. Note that error dynamics are not given in standard (canonical) Hamiltonian format as described in Skowronski [2].

To attempt to design control and adaptation laws in such a general form as shown above would be impractical and so we consider a special form of the dynamic Equations (1) and (2) which holds reasonable generality, in order to find a suitable control and adaptation law in both MRC and MRAC. We begin first with Model Reference Control (without adjustable parameters $\boldsymbol{a}$ ). 


\section{Model reference control MRC}

Let us define the sliding mode variable $s$ for our second order system:

$$
s_{i}=\dot{e}_{q_{i}}+\lambda e_{q_{i}}^{\alpha / \beta}, \quad i=1, \ldots, n,
$$

where $\alpha$ and $\beta$ are odd positive integers, $\alpha<\beta$.

Consider the traditional Lyapunov function as a quadratic form of sliding mode variables:

$$
V(\boldsymbol{s})=\frac{1}{2} \sum_{i=1}^{n} s_{i}^{2} .
$$

Denote by $E_{q_{i}}, E_{p_{i}}$ the right-hand side of the Equation (1) and by $E_{q_{m_{i}}}, E_{p_{m_{i}}}$ the right-hand side of the Equation (2). Then by definition

$$
\begin{aligned}
\dot{e}_{q_{i}} & =E_{q_{i}}-E_{q_{m_{i}}}, \\
\ddot{e}_{q_{i}} & =\dot{E}_{q_{i}}-\dot{E}_{q_{m_{i}}}, \quad i=1, \ldots, n .
\end{aligned}
$$

\subsection{Assumptions}

Reference model has its equilibria in the origin. The dynamics of the system satisfies

$$
\begin{aligned}
\dot{q}_{i} & =g_{i}(\boldsymbol{q}) p_{i}, \\
\dot{q}_{m_{i}} & =g_{m_{i}}\left(\boldsymbol{q}_{m}\right) p_{m_{i}}, \quad i=1, \ldots, n,
\end{aligned}
$$

where each $g_{i}(\cdot), g_{m_{i}}(\cdot)$, for $i=1, \ldots, n$ is a known bounded positive function.

Let us make a further assumption that $\dot{E}_{q_{i}}$ can be expressed as a linear function of $E_{p_{i}}, i=1,2$, with coefficient functions $f_{i}^{1}$ and $f_{i}^{2}$ :

$$
\dot{E}_{q_{i}}=f_{i}^{1}(\boldsymbol{q}, \boldsymbol{p}) E_{p_{i}}+f_{i}^{2}(\boldsymbol{q}, \boldsymbol{p}), \quad i=1, \ldots, n,
$$


and where each $f_{i}^{1}(\cdot)$, for $i=1, \ldots, n$ is a known bounded positive function:

$$
0 \leq\left|f_{i}^{1}(\boldsymbol{q}, \boldsymbol{p})\right| \leq B,
$$

and $B$ is a positive constant. A wide range of mechanical systems satisfy condition (6) and condition (7). Note that the control force is incorporated in $E_{p_{i}}$ terms, see Equation (1).

\subsection{Control law - MRC}

Now we calculate the time derivative of the Lyapunov function in order to extract the control law:

$$
\begin{aligned}
\dot{V}(\boldsymbol{s})= & \sum_{i=1}^{n} s_{i} \dot{s}_{i}=\sum_{i=1}^{n} s_{i}\left(\ddot{e}_{q_{i}}+\frac{\alpha}{\beta} \lambda \dot{e}_{q_{i}} e_{q_{i}}^{\alpha / \beta-1}\right) \\
= & \sum_{i=1}^{n} s_{i}\left[f_{i}^{1}(\boldsymbol{q}, \boldsymbol{p}) E_{p_{i}}+f_{i}^{2}(\boldsymbol{q}, \boldsymbol{p})-\dot{E}_{q_{m_{i}}}\right. \\
& \left.\quad+\frac{\alpha}{\beta} \lambda e_{q_{i}}^{\alpha / \beta-1}\left(E_{q_{i}}-E_{q_{m_{i}}}\right)\right] \\
= & \sum_{i=1}^{n} s_{i}\left[f_{i}^{1}(\boldsymbol{q}, \boldsymbol{p})\left(-\frac{\partial H_{p}(\boldsymbol{q}, \boldsymbol{p})}{\partial q_{i}}+Q_{i}^{D}(\boldsymbol{q}, \boldsymbol{p}, \boldsymbol{d})+Q_{i}^{F}(\boldsymbol{q}, \boldsymbol{p}, \boldsymbol{u})\right)\right. \\
& \left.\quad+f_{i}^{2}(\boldsymbol{q}, \boldsymbol{p})-\dot{E}_{q_{m_{i}}}+\frac{\alpha}{\beta} \lambda e_{q_{i}}^{\alpha / \beta-1}\left(E_{q_{i}}-E_{q_{m_{i}}}\right)\right] .
\end{aligned}
$$

The control laws are designed as follows:

$$
\begin{aligned}
Q_{i}^{F}= & \frac{\partial H_{p}}{\partial q_{i}}-Q_{i}^{D}(\boldsymbol{q}, \boldsymbol{p}, \boldsymbol{d}) \\
& -\frac{1}{f_{i}^{1}}\left(f_{i}^{2}(\boldsymbol{q}, \boldsymbol{p})-\dot{E}_{q_{m_{i}}}+\frac{\alpha}{\beta} \lambda e_{q_{i}}^{\alpha / \beta-1}\left(E_{q_{i}}-E_{q_{m_{i}}}\right)\right) \\
& -\frac{K}{f_{i}^{1}} \operatorname{sgn}\left(s_{i}\right), \quad i=1, \ldots, n,
\end{aligned}
$$


where $K>0$ is constant.

Substituting for $Q_{i}^{F}$ from the control law (10) into Equation (9), see that the control law selection makes the Lyapunov derivative semi-negative definite:

$$
\begin{aligned}
\dot{V}(\boldsymbol{s}) & =-\sum_{i=1}^{n} s_{i} K \operatorname{sgn}\left(s_{i}\right)=-K \sum_{i=1}^{n}\left|s_{i}\right| \leq-K\left(\sum_{i=1}^{n} s_{i}^{2}\right)^{1 / 2} \\
& =-\sqrt{2} K V^{1 / 2} \leq 0 .
\end{aligned}
$$

Obviously $\dot{V}=0$ only if $\boldsymbol{s}=\mathbf{0}$. This implies that $V$ reaches the sliding surface in finite time $T$. Indeed, integrating inequality (11), we find that $T$ must satisfy the inequality:

$$
T \leq t_{0}+\frac{\sqrt{2}\left(V\left(t_{0}\right)\right)^{1 / 2}}{K} .
$$

Given the expression (9) for $\dot{V}$, to show that $\boldsymbol{s} \rightarrow \mathbf{0}$ it is sufficient to show that $\dot{V} \rightarrow 0$. First, we establish that $s$ and $\dot{s}$ are bounded (that in turn shows that $\ddot{V}$ remains bounded and according to Barbalat's lemma we have $\dot{V} \rightarrow 0$ ). Given Equation (11) obviously $s$ and $\dot{s}$ are bounded, see also expression (9). Thus $s \rightarrow 0$ as $t \rightarrow \infty$. This in turn implies that error trajectories $e_{q_{i}}, i=1, \ldots, n$, tend to 0 as $t \rightarrow \infty$. Now, from (6) and the fact that $\dot{e}_{q_{i}}, i=1, \ldots, n$, tend to 0 as $t \rightarrow \infty$ (see definition of $s$ ), we see that also $e_{p_{i}}, i=1, \ldots, n$, tend to 0 as $t \rightarrow \infty$. The latter comes from

$$
\dot{e}_{q_{i}}=g_{i}(\boldsymbol{q}) p_{i}-g_{m_{i}}\left(\boldsymbol{q}_{m}\right) p_{m_{i}}, \quad i=1, \ldots, n,
$$

Because $p_{i}=p_{m_{i}}+e_{p_{i}}$ see that

$$
g_{i}(\boldsymbol{q}) e_{p_{i}}=\dot{e}_{q_{i}}-\left(g_{i}(\boldsymbol{q}) p_{i}-g_{m_{i}}\left(\boldsymbol{q}_{m}\right)\right) p_{m_{i}}, \quad i=1, \ldots, n,
$$

and this proves $e_{p_{i}}, i=1, \ldots, n$, tend to 0 as $t \rightarrow \infty$ as the equilibria of the reference model are at the origin, which requires $p_{m_{i}} \rightarrow 0$. We have shown more: namely that $\boldsymbol{s} \rightarrow \mathbf{0}$ in finite time. 


\subsection{Robot illustration - MRC}

To illustrate the method consider now the control of a cylindrical robotic manipulator [1], which has one revolute joint and two prismatic joints, see Figure 1.

The arm has length $\ell$ and its mass per unit is constant $m_{a} / \ell$. The length of the prismatic radial link changes when it slides through the hub. A force opposes the motion of the link and is modelled as a spring with an adjustable parameter $k_{s}$ (we denote it $\hat{k}_{s}$ ) which imposes zero force at $r=2 \ell / 3$. We ignore the vertical motion along the hub. Denote $q_{1}=r$ and $q_{2}=\theta$. The Hamiltonian describing the dynamics of the manipulator is

$$
H(q, p)=\frac{1}{2}\left(\frac{1}{m_{a}+m_{\ell}} p_{1}^{2}+\frac{1}{C\left(q_{1}\right)} p_{2}^{2}+k_{s}\left(q_{1}-\frac{2}{3} \ell\right)^{2}\right),
$$

where $C(q)=\frac{m_{a}}{4 \ell}\left(q^{3}+(\ell-q)^{3}\right)+m_{\ell} q^{2}+I$; and $I$ is the effective moment of inertia of the rotating masses excluding $m_{a}$ and $m_{\ell}$. We introduce the canonical transformation $(\boldsymbol{q}, \boldsymbol{p}) \rightarrow(\boldsymbol{Q}, \boldsymbol{P})$.

$$
\begin{gathered}
q_{1}=\frac{Q_{1}}{\sqrt{m_{a}+m_{\ell}}}+\frac{2}{3} \ell ; \quad p_{1}=\sqrt{m_{a}+m_{\ell}} P_{1} ; \\
q_{2}=Q_{2} ; \quad p_{2}=P_{2} .
\end{gathered}
$$

After the canonical transformation the Hamiltonian

$$
H(Q, P)=\frac{1}{2}\left(P_{1}^{2}+\frac{1}{C\left(Q_{1} / \sqrt{m_{a}+m_{\ell}}+\frac{2}{3} \ell\right)} P_{2}^{2}+\frac{k_{s}}{m_{a}+m_{\ell}} Q_{1}^{2}\right) .
$$

Without loss of generality, we return to the original notation keeping in mind that $(p, q)$ denotes now the new coordinates $(P, Q)$. The dynamics of the manipulator are now

$$
\dot{q}_{1}=p_{1},
$$


(a)

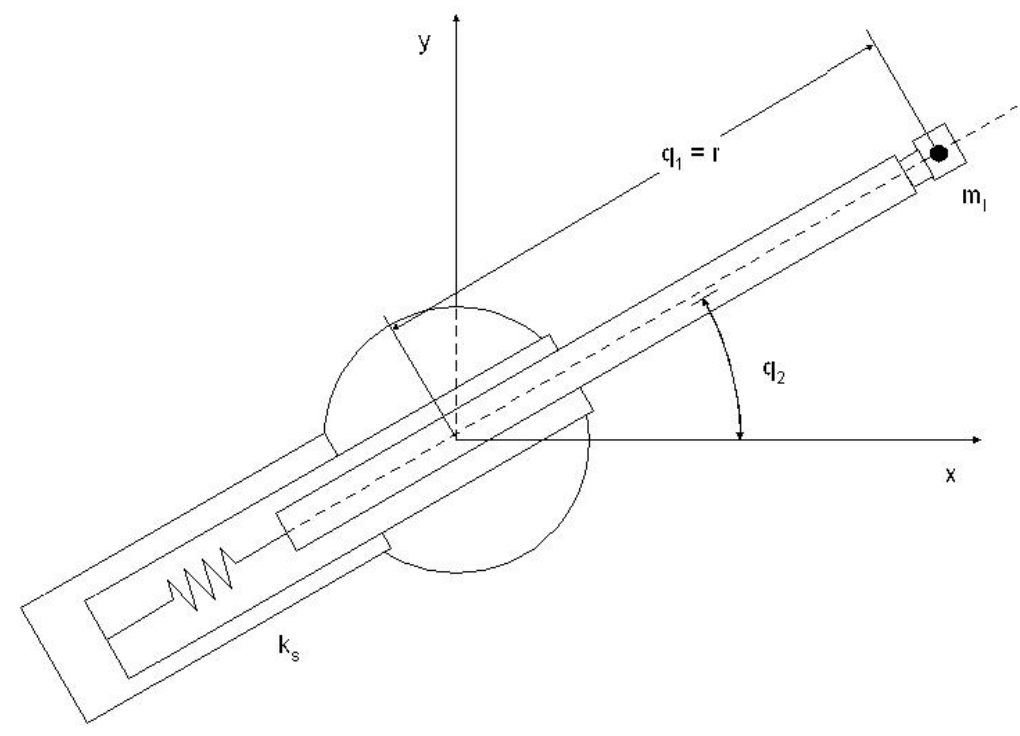

(b)

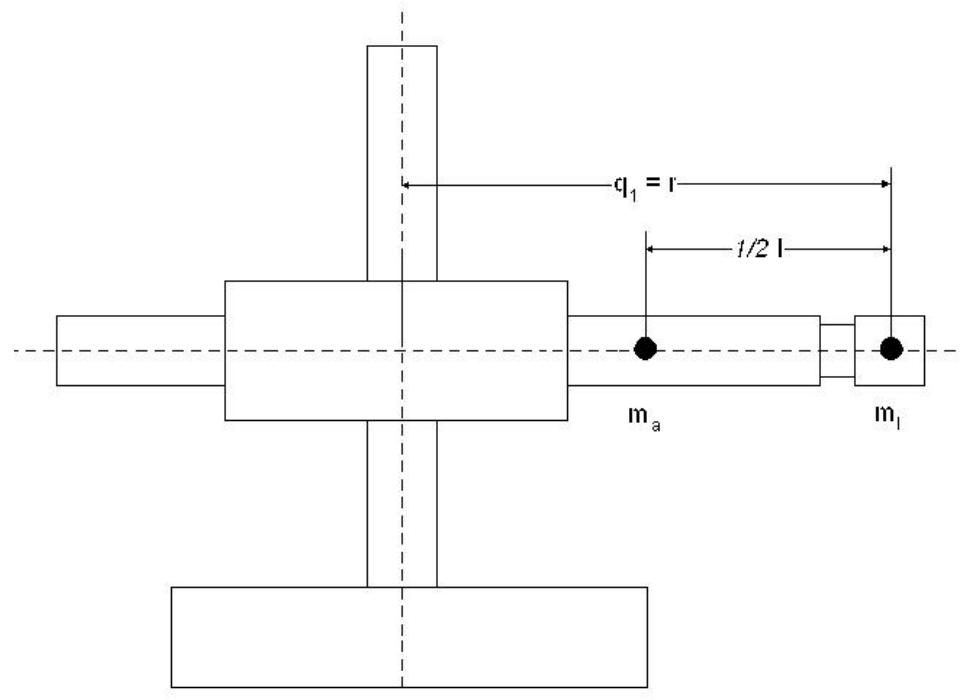

Figure 1: Cylindrical robot manipulator: (a) top view; (b) side view. 


$$
\begin{aligned}
\dot{q}_{2}= & \frac{p_{2}}{C\left(q_{c}\right)}, \\
\dot{p}_{1}= & {\left[\left(\frac{3}{4} m_{a}+m_{\ell}\right) \frac{q_{1}}{m_{a}+m_{\ell}}\right.} \\
& \left.\quad+\left(\frac{1}{8} m_{a}+\frac{2}{3} m_{\ell}\right) \frac{\ell}{\sqrt{m_{a}+m_{\ell}}}\right]\left(\frac{p_{2}}{C\left(q_{c}\right)}\right)^{2} \\
& \quad-\frac{k_{s}}{\left(m_{a}+m_{\ell}\right)} q_{1}+Q_{1}^{D}+Q_{1}^{F}, \\
\dot{p}_{2}= & Q_{2}^{D}+Q_{2}^{F},
\end{aligned}
$$

where

$$
\begin{aligned}
C\left(q_{c}\right) & =\frac{m_{a}}{4 \ell}\left(q_{c}^{3}+\left(\ell-q_{c}\right)^{3}\right)+m_{l} q_{c}^{2}+I, \\
q_{c} & =\frac{q_{1}}{\sqrt{m_{a}+m_{\ell}}}+\frac{2}{3} \ell .
\end{aligned}
$$

Damping forces are defined by $Q_{i}^{D}(\boldsymbol{q}, \boldsymbol{p})=-d_{i} p_{i}, i=1,2$, and $d_{i}$ are the positive damping coefficients.

Consider a reference model with dynamics

$$
\begin{aligned}
& \dot{q}_{m_{1}}=p_{m_{1}} \\
& \dot{q}_{m_{2}}=p_{m_{2}}, \\
& \dot{p}_{m_{1}}=-\frac{k_{s_{m}}}{m_{m_{a}}+m_{m_{\ell}}} q_{m_{1}}+Q_{m_{1}}^{D}+Q_{m_{1}}^{F}, \\
& \dot{p}_{m_{2}}=-s_{m} q_{m_{2}}+Q_{m_{2}}^{D}+Q_{m_{2}}^{F},
\end{aligned}
$$

where analogously $Q_{m_{i}}^{D}\left(\boldsymbol{q}_{m}, \boldsymbol{p}_{m}\right)=-d_{m_{i}} p_{m_{i}}, i=1,2$, and $d_{m_{i}}$ are positive damping coefficients. The term $s_{m} q_{m_{2}}$ represents spring forces. The equilibria of the model coincide with the original system, except that $q_{m_{2}}^{e}=0$, unlike the systems' $q_{2}^{e}$ that can be arbitrary.

With the given Lyapunov function (4), we find its time derivative

$$
\dot{V}=s_{1} \dot{s}_{1}+s_{2} \dot{s}_{2}
$$




$$
=s_{1}\left(\ddot{e}_{q_{1}}+\frac{\alpha}{\beta} \lambda e_{q_{1}}^{\alpha / \beta-1} \dot{e}_{q_{1}}\right)+s_{2}\left(\ddot{e}_{q_{2}}+\frac{\alpha}{\beta} \lambda e_{q_{2}}^{\alpha / \beta-1} \dot{e}_{q_{2}}\right) .
$$

In this case

$$
\dot{e}_{q_{1}}=\dot{q}_{1}-\dot{q}_{m_{1}}=p_{1}-p_{m_{1}}=e_{p_{1}} \quad \dot{e}_{q_{2}}=\frac{p_{2}}{C\left(q_{c}\right)}-p_{m_{2}} .
$$

Now we calculate $\dot{C}\left(q_{c}\right)$ noting that $\dot{q}_{c}=\frac{\dot{q}_{1}}{\sqrt{m_{a}+m_{\ell}}}$.

$$
\begin{aligned}
\dot{C}\left(q_{c}\right)= & \frac{m_{a}}{4 \ell}\left(3 q_{c}^{2} \dot{q}_{c}-3\left(\ell-q_{c}\right)^{2} \dot{q}_{c}\right)+2 m_{\ell} q_{c} \dot{q}_{c} \\
= & \frac{3}{4} \frac{m_{a} \dot{q}_{1}}{\sqrt{m_{a}+m_{\ell}}}\left(\frac{2 q_{1}}{\sqrt{m_{a}+m_{\ell}}}+\frac{1}{3} \ell\right) \\
& +\frac{2 m_{\ell} q_{1} \dot{q}_{1}}{m_{a}+m_{\ell}}+\frac{4 m_{\ell} \ell \dot{q}_{1}}{3 \sqrt{m_{a}+m_{\ell}}} .
\end{aligned}
$$

In the above formula $\dot{q}_{1}$ can be replaced by $p_{1}$. For notational convenience, denote by $E_{i}$ the right-hand side of the system Equations (13), and by $M_{i}$ the right-hand side of the model (15), $i=1, \ldots, 4$. Then

$$
\begin{aligned}
\dot{e}_{q_{i}} & =E_{i}-M_{i}, \quad i=1,2 \\
\dot{e}_{p_{j-2}} & =E_{j}-M_{j}, \quad j=3,4 \\
\ddot{e}_{q_{1}} & =E_{3}-M_{3}, \\
\ddot{e}_{q_{2}} & =\dot{E}_{2}-M_{4},
\end{aligned}
$$

where

$$
\dot{E}_{2}=\frac{C\left(q_{c}\right) \dot{p}_{2}-\dot{C}\left(q_{c}\right) p_{2}}{C^{2}\left(q_{c}\right)} .
$$

Now, rewrite the expression (9) for $\dot{V}$ as

$$
\begin{aligned}
\dot{V}(s)= & s_{1}\left(E_{3}-M_{3}+\frac{\alpha}{\beta} \lambda e_{q_{1}}^{\alpha / \beta-1} e_{p_{1}}\right) \\
& +s_{2}\left(\dot{E}_{2}-M_{4} \frac{\alpha}{\beta} \lambda e_{q_{2}}^{\alpha / \beta-1}\left(E_{2}-M_{2}\right)\right)
\end{aligned}
$$




$$
\begin{aligned}
= & s_{1}\left(E_{3}-M_{3}+\frac{\alpha}{\beta} \lambda e_{q_{1}}^{\alpha / \beta-1} e_{p_{1}}\right) \\
& +s_{2}\left(\frac{E_{4}}{C\left(q_{c}\right)}-\frac{\dot{C}\left(q_{c}\right) p_{2}}{C^{2}\left(q_{c}\right)}-M_{4}+\frac{\alpha}{\beta} \lambda e_{q_{2}}^{\alpha / \beta-1}\left(E_{2}-M_{2}\right)\right) .
\end{aligned}
$$

\subsection{Control law for robotic example — MRC}

The control force is now incorporated in $E_{p_{1}}$ and $E_{p_{2}}$-terms. We identify, from (9) and (17), the following terms in (10)

$$
f_{1}^{1}=1.0, \quad f_{1}^{2}=0, \quad f_{2}^{1}=\frac{1}{C\left(q_{c}\right)}, \quad f_{2}^{2}=-\frac{\dot{C}\left(q_{c}\right)}{C^{2}\left(q_{c}\right)} p_{2},
$$

and

$$
\begin{aligned}
& E_{q_{m_{1}}}=p_{m_{1}}, \\
& E_{q_{m_{2}}}=p_{m_{2}}, \\
& \dot{E}_{q_{m_{1}}}=E_{p_{m_{1}}}=-\frac{k_{s_{m}}}{m_{m_{a}}+m_{m_{\ell}}} q_{m_{1}}+Q_{m_{1}}^{D}+Q_{m_{1}}^{F}, \\
& \dot{E}_{q_{m_{2}}}=E_{p_{m_{2}}}=-s_{m} q_{m_{2}}+Q_{m_{2}}^{D}+Q_{m_{2}}^{F} .
\end{aligned}
$$

Note that

$$
\begin{aligned}
\frac{\partial H_{p}}{\partial q_{1}}=- & {\left[\left(\frac{3}{4} m_{a}+m_{\ell}\right) \frac{q_{1}}{\left(m_{a}+m_{\ell}\right)}\right.} \\
& \left.+\left(\frac{1}{8} m_{a}+\frac{2}{3} m_{\ell}\right) \frac{\ell}{\sqrt{m_{a}+m_{\ell}}}\right]\left(\frac{p_{2}}{C\left(q_{c}\right)}\right)^{2} \\
& +\frac{k_{s}}{\left(m_{a}+m_{\ell}\right)} q_{1}, \\
\frac{\partial H_{p}}{\partial q_{2}}= & 0 .
\end{aligned}
$$


Then the control laws according to (10) are:

$$
\begin{aligned}
Q_{1}^{F}=- & {\left[\left(\frac{3}{4} m_{a}+m_{\ell}\right) \frac{q_{1}}{\left(m_{a}+m_{\ell}\right)}\right.} \\
& \left.+\left(\frac{1}{8} m_{a}+\frac{2}{3} m_{\ell}\right) \frac{\ell}{\sqrt{m_{a}+m_{\ell}}}\right]\left(\frac{p_{2}}{C\left(q_{c}\right.}\right)^{2} \\
+ & \frac{k_{s}}{\left(m_{a}+m_{\ell}\right)} q_{1}-Q_{1}^{D}-\frac{1}{f_{1}^{1}}\left(-E_{p_{m_{1}}}+\frac{\alpha}{\beta} \lambda e_{q_{1}}^{\alpha / \beta-1} e_{p_{1}}\right) \\
& -\frac{K}{f_{1}^{1}} \operatorname{sgn}\left(s_{1}\right), \\
Q_{2}^{F}= & -Q_{2}^{D}-\frac{1}{f_{2}^{1}}\left(-E_{p_{m_{2}}}-\frac{\dot{C}\left(q_{c}\right) p_{2}}{C^{2}\left(q_{c}\right)}+\frac{\alpha}{\beta} \lambda e_{q_{2}}^{\alpha / \beta-1}\left(E_{q_{2}}-E_{q_{m_{2}}}\right)\right) \\
& -\frac{K}{f_{2}^{1}} \operatorname{sgn}\left(s_{2}\right) .
\end{aligned}
$$

Substituting the control law (20) and (21) into Equation (17) we obtain:

$$
\begin{aligned}
\dot{V} & =-s_{1} K \operatorname{sgn}\left(s_{1}\right)-s_{2} K \operatorname{sgn}\left(s_{2}\right)=-K\left(\left|s_{1}\right|+\left|s_{2}\right|\right) \\
& \leq-K\left(s_{1}^{2}+s_{2}^{2}\right)^{1 / 2}=-\sqrt{2} K V^{1 / 2} \leq 0 .
\end{aligned}
$$

In our computer simulations we assumed the following parameter values for the plant and model: $m_{a}=10.0, m_{m_{a}}=10.5, m_{\ell}=1.25, m_{m_{\ell}}=1.5$, $\ell=1.0, \ell_{m}=1.3, I=1.0, k_{s}=100.0, k_{s_{m}}=110.0, d_{1}=5.0, d_{m_{1}}=6.5$, $d_{2}=0.001, d_{m_{2}}=0.8$, and $s_{m}=9.1$. The values for the constants were chosen as: $K=4, \lambda=2, \alpha=3$ and $\beta=5$. The initial conditions: $q_{1}=0.1, q_{2}=3.0, p_{1}=0.0, p_{2}=0.0, q_{m_{1}}=0.6, q_{m_{2}}=0.1, p_{m_{1}}=4.0$, and $p_{m_{2}}=3.2$.

The error trajectory and controller time history for the simulations are shown on Figure 2. The time history of the Lyapunov and its derivative, together with the sliding mode variables are shown in Figure 3. 


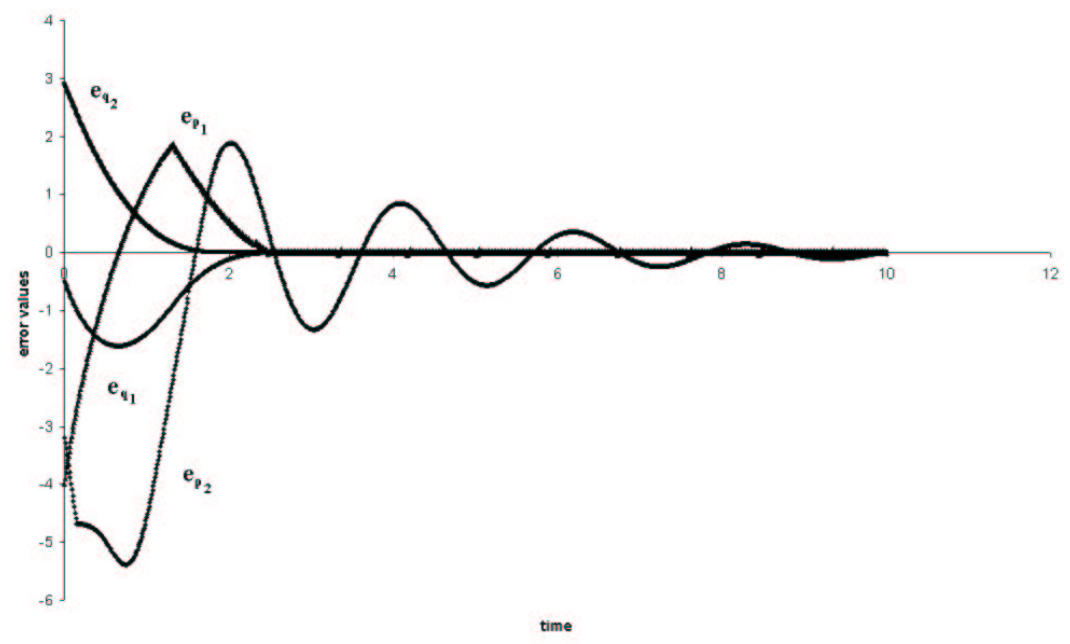

(a)

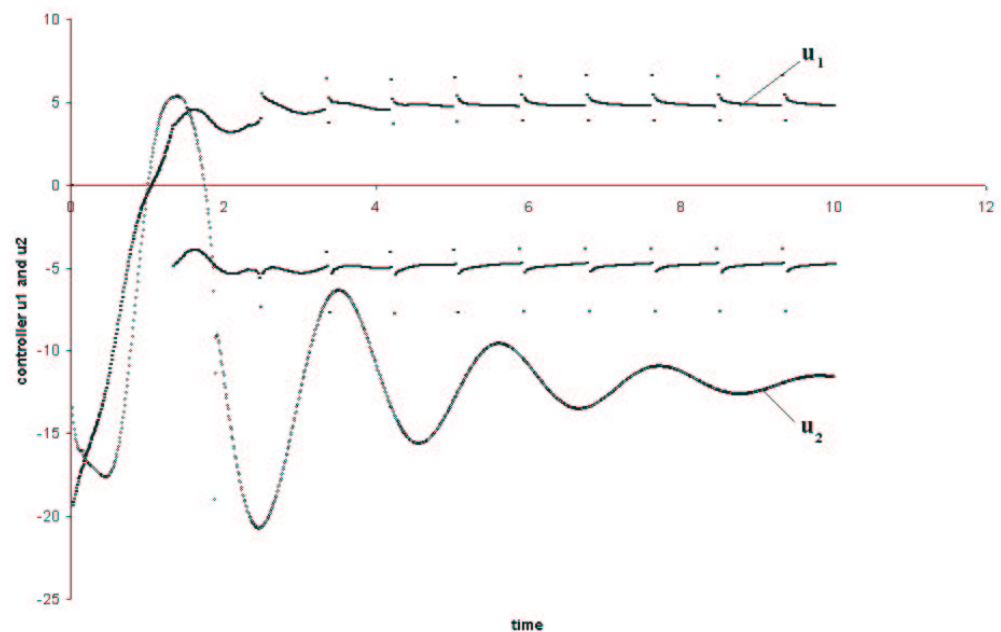

(b)

Figure 2: (a) Error convergence for MRC (b) Controller for MRC 


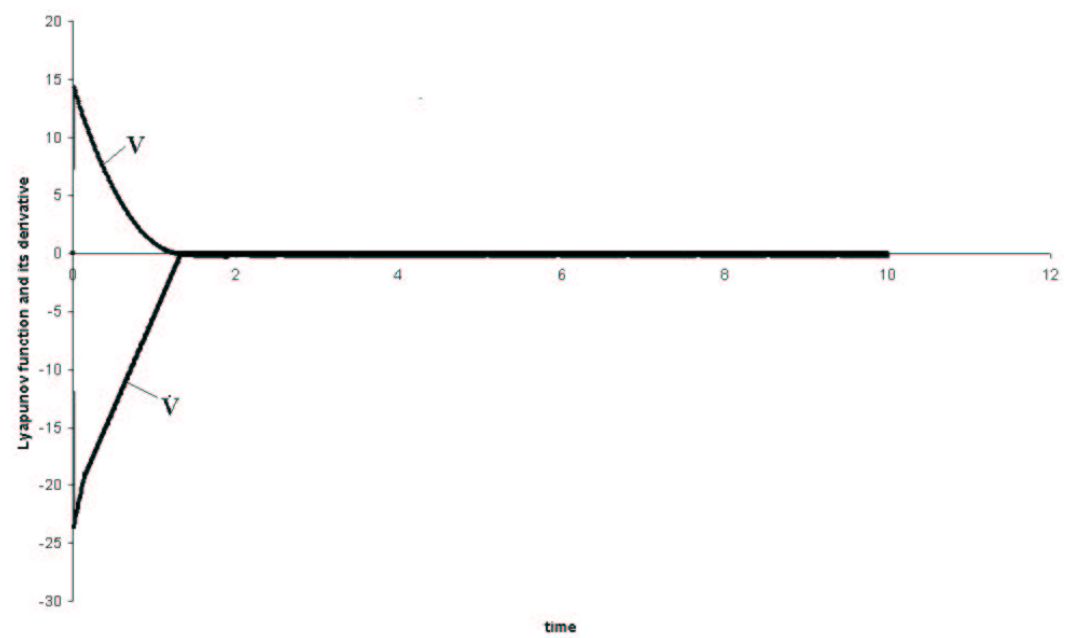

(a)

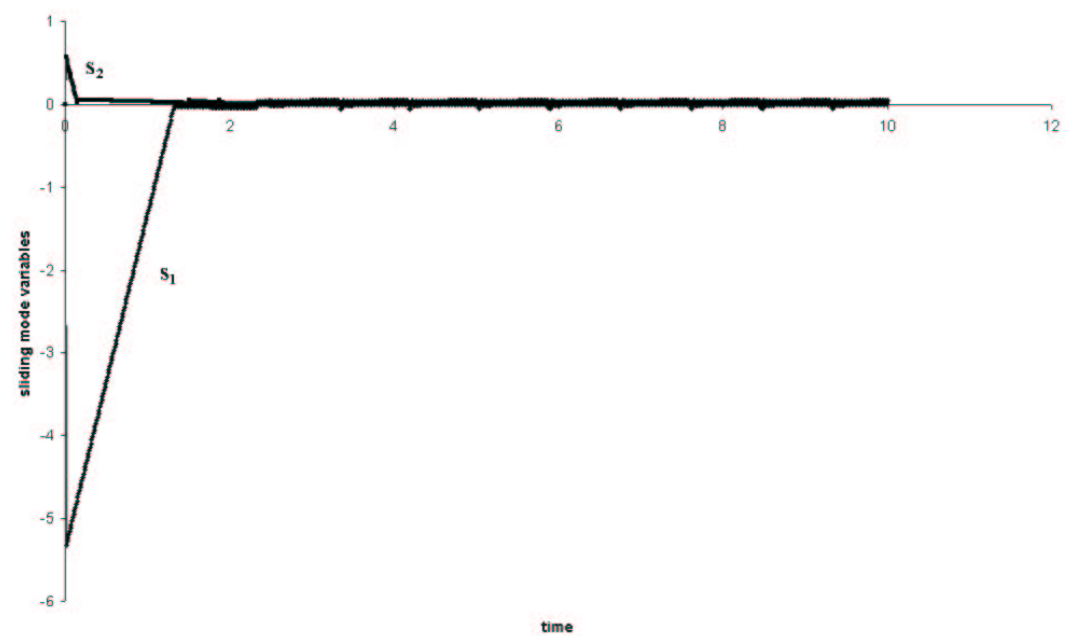

(b)

Figure 3: (a) Lyapunov function and its derivative (b) Sliding mode variables 
The convergence times of the error trajectories to within an $\epsilon$-envelope of the origin (which is defined as a set of all states that lie within distance of $\epsilon$ from the origin) are: $T_{c}=9.47$ for $\epsilon=0.1$ and $T_{c}=9.74$ for $\epsilon=0.05$.

\section{Model reference adaptive control}

\section{MRAC}

Similarly we define a Lyapunov function for the adaptive case as a quadratic form of sliding mode variables plus its adaptive terms:

$$
V(\boldsymbol{s}, \boldsymbol{a}, \boldsymbol{d})=\frac{1}{2} \sum_{i=1}^{n} s_{i}^{2}+\frac{1}{2 \gamma} \sum_{i=1}^{n} \tilde{a}_{i}^{2}+\frac{1}{2 \gamma} \sum_{i=1}^{n} \tilde{d}_{i}^{2},
$$

where $\gamma>0$ is constant and

$$
\tilde{a}_{i}=\hat{a}_{i}-a_{i}^{*}, \quad \tilde{d}_{i}=\hat{d}_{i}-d_{i}^{*},
$$

and where $\hat{a}_{i}$ and $\hat{d}_{i}$ are our estimates of uncertain parameters whereas $a_{i}^{*}$ and $d_{i}^{*}$ are their true values (but they may be unknown). We assume that condition (7) holds for MrAC. Again we calculate the time-derivative of Lyapunov function in order to extract the control law:

$$
\begin{aligned}
\dot{V}(\boldsymbol{s}, \boldsymbol{a}, \boldsymbol{d})= & \sum_{i=1}^{n}\left[s_{i} \dot{s}_{i}+\frac{1}{\gamma} \dot{\tilde{a}}_{i} \tilde{a}_{i}+\frac{1}{\gamma} \dot{\tilde{d}}_{i} \tilde{d}_{i}\right] \\
= & \sum_{i=1}^{n}\left[s_{i}\left(\ddot{e}_{q_{i}}+\frac{\alpha}{\beta} \lambda \dot{e}_{q_{i}} e_{q_{i}}^{\alpha / \beta-1}\right)+\frac{1}{\gamma} \dot{\tilde{a}}_{i} \tilde{a}_{i}+\frac{1}{\gamma} \dot{\tilde{d}}_{i} \tilde{d}_{i}\right] \\
= & \sum_{i=1}^{n}\left[s _ { i } \left(f_{i}^{1}(\boldsymbol{q}, \boldsymbol{p}) E_{p_{i}}+f_{i}^{2}(\boldsymbol{q}, \boldsymbol{p})-\dot{E}_{q_{m_{i}}}\right.\right. \\
& \left.\left.\quad+\frac{\alpha}{\beta} \lambda e_{q_{i}}^{\alpha / \beta-1}\left(E_{q_{i}}-E_{q_{m_{i}}}\right)\right)+\frac{1}{\gamma} \dot{\tilde{\tilde{a}}}_{i} \tilde{a}_{i}+\frac{1}{\gamma} \dot{\tilde{d}}_{i} \tilde{d}_{i}\right] \\
= & \sum_{i=1}^{n}\left[f _ { i } ^ { 1 } ( \boldsymbol { q } , \boldsymbol { p } ) \left(-\frac{\partial H_{p}(\boldsymbol{q}, \boldsymbol{p}, \boldsymbol{a})}{\partial q_{i}}+Q_{i}^{D}(\boldsymbol{q}, \boldsymbol{p}, \boldsymbol{d})\right.\right.
\end{aligned}
$$




$$
\begin{aligned}
& \left.+Q_{i}^{F}(\boldsymbol{q}, \boldsymbol{p}, \boldsymbol{a}, \boldsymbol{u})\right)+f_{i}^{2}(\boldsymbol{q}, \boldsymbol{p})-\dot{E}_{q_{m_{i}}} \\
+ & \left.\frac{\alpha}{\beta} \lambda e_{q_{i}}^{\alpha / \beta-1}\left(E_{q_{i}}-E_{q_{m_{i}}}\right)\right]+\frac{1}{\gamma} \sum_{i=1}^{n}\left(\dot{\tilde{\tilde{a}}}_{i} \tilde{a}_{i}+\dot{\tilde{d}}_{i} \tilde{d}_{i}\right) .
\end{aligned}
$$

We assume that plant dynamics can be linearly parametrised in terms of the unknown parameters $a_{i}$. Note: the damping force $Q_{i}^{D}$ (for all practical purposes) is linear in its damping coefficient. Let us denote

$$
\begin{aligned}
\frac{\partial H_{p}(\boldsymbol{q}, \boldsymbol{p}, \boldsymbol{a})}{\partial q_{i}} & =a_{i} \frac{\partial H_{p}^{\ell}(\boldsymbol{q}, \boldsymbol{p})}{\partial q_{i}}, \\
Q_{i}^{D}(\boldsymbol{q}, \boldsymbol{p}, \boldsymbol{d}) & =d_{i} Q_{i}^{D \ell}(\boldsymbol{q}, \boldsymbol{p})
\end{aligned}
$$

where superscript $\ell$ indicates function after linear parametrisation.

\subsection{Control and adaptation law in MRAC}

We design the control law in MRAC version:

$$
\begin{aligned}
Q_{i}^{F}= & \hat{a}_{i} \frac{\partial H_{p}^{\ell}}{\partial q_{i}}-\hat{d}_{i} Q_{i}^{D \ell}(\boldsymbol{q}, \boldsymbol{p}) \\
& -\frac{1}{f_{i}^{1}}\left(-\dot{E}_{q_{m_{i}}}+\frac{\alpha}{\beta} \lambda e_{q_{i}}^{\alpha / \beta-1}\left(E_{q_{i}}-E_{q_{m_{i}}}\right)\right) \\
& -f_{i}^{2}(\boldsymbol{q}, \boldsymbol{p})-\frac{K}{f_{i}^{1}} \operatorname{sgn}\left(s_{i}\right), \quad i=1, \ldots, n .
\end{aligned}
$$

After substituting from Equation (25) into Equation (23) we obtain

$$
\begin{aligned}
\dot{V}(\boldsymbol{s}, \boldsymbol{a}, \boldsymbol{d})= & \sum_{i=1}^{n} s_{i}\left[\left(\hat{a}_{i}-a_{i}\right) \frac{\partial H_{p}^{\ell}}{\partial q_{i}}-\left(\hat{d}_{i}-d_{i}\right) Q_{i}^{D \ell}(\boldsymbol{q}, \boldsymbol{p})\right] \\
& -\sum_{i=1}^{n} s_{i} K \operatorname{sgn}\left(s_{i}\right)+\frac{1}{\gamma} \sum_{i=1}^{n}\left(\dot{\tilde{a}}_{i} \tilde{a}_{i}+\dot{\tilde{d}}_{i} \tilde{d}_{i}\right)
\end{aligned}
$$




$$
\begin{aligned}
= & \sum_{i=1}^{n}\left[\tilde{a}_{i}\left(s_{i} \frac{\partial H_{p}^{\ell}}{\partial q_{i}}+\frac{1}{\gamma} \dot{\hat{a}}_{i}\right)+\tilde{d}_{i}\left(-s_{i} Q_{i}^{D \ell}(\boldsymbol{q}, \boldsymbol{p})+\frac{1}{\gamma} \dot{\hat{d}}_{i}\right)\right] \\
& -\sum_{i=1}^{n} s_{i} K \operatorname{sgn}\left(s_{i}\right) .
\end{aligned}
$$

We now define the adaptation law

$$
\begin{aligned}
& \dot{\hat{a}}_{i}=-\frac{K \gamma}{\sqrt{\gamma}} \operatorname{sgn}\left(\tilde{a}_{i}\right)-\gamma s_{i} \frac{\partial H_{p}^{\ell}}{\partial q_{i}}-\frac{\gamma K}{\sqrt{\gamma}} \operatorname{sgn}\left(\tilde{a}_{i}\right), \\
& \dot{\hat{d}}_{i}=-\frac{K \gamma}{\sqrt{\gamma}} \operatorname{sgn}\left(\tilde{d}_{i}\right)+\gamma s_{i} Q_{i}^{D \ell}-\frac{\gamma K}{\sqrt{\gamma}} \operatorname{sgn}\left(\tilde{d}_{i}\right) .
\end{aligned}
$$

\subsection{Convergence}

Now we can return to our evaluation of $\dot{V}$ and prove it to be negative semidefinite, and consequently securing asymptotic tracking convergence. Substituting control (25) and adaptation laws (27) and (28) into Equation (26), and using a simple algebraic inequality [4], we obtain

$$
\begin{aligned}
\dot{V}(\boldsymbol{s}, \boldsymbol{a}, \boldsymbol{d}) & =-K \sum_{i=1}^{n}\left[\frac{1}{\sqrt{\gamma}} \tilde{a}_{i} \operatorname{sgn}\left(\tilde{a}_{i}\right)+\frac{1}{\sqrt{\gamma}} \tilde{d}_{i} \operatorname{sgn}\left(\tilde{d}_{i}\right)+s_{i} \operatorname{sgn}\left(s_{i}\right)\right] \\
& \left.=-K \sum_{i=1}^{n}\left[\frac{1}{\sqrt{\gamma}}\left|\tilde{a}_{i}\right|+\frac{1}{\sqrt{\gamma}}\left|\tilde{d}_{i}\right|\right)+s_{i} \operatorname{sgn}\left(s_{i}\right)\right] \\
& =-K \sum_{i=1}^{n}\left|s_{i}\right| \leq-K\left(\sum_{i=1}^{n} s_{i}^{2}\right)^{1 / 2} \\
& \leq-\sqrt{2} K V^{1 / 2} \leq 0 .
\end{aligned}
$$

Obviously $\dot{V}=0$ only if $\boldsymbol{s}=\mathbf{0}$. This implies that $V$ reaches the origin in a finite time $T$, that is, $V(T)=0$; indeed, integrating inequality (29) see that

$$
T \leq t_{0}+\frac{\sqrt{2} V^{1 / 2}\left(t_{0}\right)}{K} .
$$


Analogously, as in MRC section, we show that given the expression (23) we have $\boldsymbol{s} \rightarrow \mathbf{0}$. It is sufficient to show that $\dot{V} \rightarrow 0$. Clearly $s$ and $\dot{s}$ are bounded, this in turn shows that $\ddot{V}$ remains bounded and according to Barbalat's lemma we have $\dot{V} \rightarrow 0$. Given Equation (29) obviously $s$ and $\dot{s}$ are bounded, see also Equation (23). Thus $s \rightarrow 0$ as $t \rightarrow \infty$. This in turn implies that error trajectories $e_{q_{i}}, i=1, \ldots, n$, tend to 0 as $t \rightarrow \infty$. By the same argument as in MRC section we can show that also $e_{p_{i}}, i=1, \ldots, n$, tend to 0 as $t \rightarrow \infty$. And again $\boldsymbol{s} \rightarrow \mathbf{0}$ in finite time, see Equation (30).

\subsection{Robot illustration - MRAC}

We consider the same example of robotic manipulator as in the MRC example but now we consider the damping forces as uncertain due to unknown damping coefficients. Damping forces are defined by $Q_{i}^{D}(\boldsymbol{q}, \boldsymbol{p}, \boldsymbol{d})=-d_{i} p_{i}$, $i=1,2$, and $d_{i}$ are the positive damping coefficients of unknown value, and thus replaced in our control law by adjustable damping coefficients $\hat{d}_{1}$ and $\hat{d}_{2}$. Similarly $k_{s}$ is of uncertain value and thus subject to adaptation mechanism.

We modify the previous Lyapunov function to introduce adaptive terms

$$
V\left(\boldsymbol{s}, d_{1}, d_{2}, k_{s}\right)=\sum_{i=1}^{2} s_{i}^{2}+\frac{1}{2 \gamma} \tilde{k}_{s}^{2}+\frac{1}{2 \gamma}\left(\tilde{d}_{1}^{2}+\tilde{d}_{2}^{2}\right) .
$$

Its time derivative is

$$
\begin{aligned}
\dot{V}= & s_{1} \dot{s}_{1}+s_{2} \dot{s}_{2}+\frac{1}{\gamma} \dot{\tilde{k}}_{s} \tilde{k}_{s}+\frac{1}{\gamma} \sum_{i=1}^{2} \dot{\tilde{d}}_{i} \tilde{d}_{i}=s_{1}\left(\ddot{e}_{q_{1}}+\frac{\alpha}{\beta} \lambda e_{q_{1}}^{\alpha / \beta-1} \dot{e}_{q_{1}}\right) \\
& +s_{2}\left(\ddot{e}_{q_{2}}+\frac{\alpha}{\beta} \lambda e_{q_{2}}^{\alpha / \beta-1} \dot{e}_{q_{2}}\right)+\frac{1}{\gamma} \dot{\tilde{k}}_{s} \tilde{k}_{s}+\frac{1}{\gamma} \sum_{i=1}^{2} \dot{\tilde{d}}_{i} \tilde{d}_{i} .
\end{aligned}
$$

Now, rewrite the expression for $\dot{V}$ using definitions and notation from the MRC section:

$$
\dot{V}(s)=s_{1}\left(E_{p_{1}}-E_{p_{m_{1}}}+\frac{\alpha}{\beta} \lambda e_{q_{1}}^{\alpha / \beta-1} e_{p_{1}}\right)
$$




$$
\begin{aligned}
& +s_{2}\left(\dot{E}_{q_{2}}-E_{p_{m_{2}}} \frac{\alpha}{\beta} \lambda e_{q_{2}}^{\alpha / \beta-1}\left(E_{q_{2}}-E_{q_{m_{2}}}\right)\right) \\
& +\frac{1}{\gamma} \dot{\tilde{k}}_{s} \tilde{k}_{s}+\frac{1}{\gamma} \sum_{i=1}^{2} \dot{\tilde{d}}_{i} \tilde{d}_{i} \\
= & s_{1}\left(E_{p_{1}}-E_{p_{m_{1}}}+\frac{\alpha}{\beta} \lambda e_{q_{1}}^{\alpha / \beta-1} e_{p_{1}}\right) \\
& +s_{2}\left(\frac{E_{p_{2}}}{C\left(q_{c}\right)}-\frac{\dot{C}\left(q_{c}\right) p_{2}}{C^{2}\left(q_{c}\right)}-E_{p_{m_{2}}}+\frac{\alpha}{\beta} \lambda e_{q_{2}}^{\alpha / \beta-1}\left(E_{q_{2}}-E_{q_{m_{2}}}\right)\right) \\
& +\frac{1}{\gamma} \dot{\tilde{k}}_{s} \tilde{k}_{s}+\frac{1}{\gamma} \sum_{i=1}^{2} \dot{\tilde{d}}_{i} \tilde{d}_{i} .
\end{aligned}
$$

\subsection{Control and adaptation law}

Again, the control force is incorporated in $E_{p_{1}}$ and $E_{p_{2}}$-terms. According to (25) our control law is

$$
\begin{aligned}
Q_{1}^{F}=- & {\left[\left(\frac{3}{4} m_{a}+m_{\ell}\right) \frac{q_{1}}{m_{a}+m_{\ell}}\right.} \\
& \left.+\left(\frac{1}{8} m_{a}+\frac{2}{3} m_{\ell}\right) \frac{\ell}{\sqrt{m_{a}+m_{\ell}}}\right]\left(\frac{p_{2}}{C\left(q_{c}\right.}\right)^{2} \\
+ & \frac{\hat{k}_{s}}{\left(m_{a}+m_{\ell}\right)} q_{1}-\hat{Q}_{1}^{D}-\frac{1}{f_{1}^{1}}\left(-E_{p_{m_{1}}}+\frac{\alpha}{\beta} \lambda e_{q_{1}}^{\alpha / \beta-1} e_{p_{1}}\right) \\
& -\frac{K}{f_{1}^{1}} \operatorname{sgn}\left(s_{1}\right), \\
Q_{2}^{F}= & -\hat{Q}_{2}^{D} \frac{1}{f_{2}^{1}}\left(-E_{p_{m_{2}}}-\frac{\dot{C}\left(q_{c}\right) p_{2}}{C^{2}\left(q_{c}\right)}+\frac{\alpha}{\beta} \lambda e_{q_{2}}^{\alpha / \beta-1}\left(E_{q_{2}}-E_{q_{m_{2}}}\right)\right) \\
& -\frac{K}{f_{2}^{1}} \operatorname{sgn}\left(s_{2}\right),
\end{aligned}
$$


where $K$ is a positive constant, and $\hat{k}_{s}, \hat{Q}_{i}^{D \ell}=\hat{d}_{i} Q_{i}^{D \ell}, i=1,2$, are our estimates of uncertain functions. Other terms are defined as in MRC case.

Substituting the control law (33) and (34) into Equation (32):

$$
\begin{aligned}
\dot{V}= & s_{1} \tilde{k}_{s} \frac{q_{1}}{m_{a}+m_{\ell}}+s_{1} \tilde{d}_{1} p_{1}+s_{2} \tilde{d}_{2} \frac{p_{2}}{C\left(q_{c}\right)} \\
& -s_{1} K \operatorname{sgn}\left(s_{1}\right)-s_{2} K \operatorname{sgn}\left(s_{2}\right)+\frac{1}{\gamma} \dot{\hat{k}}_{s} \tilde{k}_{s}+\frac{1}{\gamma} \sum_{i=1}^{2} \dot{\hat{d}}_{i} \tilde{d}_{i} .
\end{aligned}
$$

Then we formulate our adaptation law:

$$
\begin{aligned}
& \dot{\hat{d}}_{1}=-\frac{K \gamma}{\sqrt{\gamma}}-\gamma s_{1} p_{1}, \\
& \dot{\hat{d}}_{2}=-\frac{K \gamma}{\sqrt{\gamma}}-\gamma s_{2} \frac{p_{2}}{C\left(q_{c}\right)}, \\
& \dot{\hat{k}}_{s}=-\frac{K \gamma}{\sqrt{\gamma}}-\gamma s_{1} \frac{q_{1}}{m_{a}+m_{\ell}} .
\end{aligned}
$$

Then after substituting from Equation (36) into Equation (35) see that

$$
\begin{aligned}
\dot{V}= & s_{1} \tilde{k}_{s} \frac{q_{1}}{m_{a}+m_{\ell}}+s_{1} \tilde{d}_{1} p_{1}+s_{2} \tilde{d}_{2} \frac{p_{2}}{C\left(q_{c}\right)}-s_{1} K \operatorname{sgn}\left(s_{1}\right) \\
& -s_{2} K \operatorname{sgn}\left(s_{2}\right)+\frac{1}{\gamma}\left[-\gamma s_{1} \frac{q_{1}}{m_{a}+m_{\ell}}\right] \tilde{k}_{s} \\
& +\frac{1}{\gamma}\left[-\gamma s_{1} p_{1}\right] \tilde{d}_{1}+\frac{1}{\gamma}\left[-\gamma s_{2} \frac{p_{2}}{C\left(q_{c}\right)}\right] \tilde{d}_{2} \\
= & -K\left(\left|s_{1}\right|+\left|s_{2}\right|\right) \leq-K\left(s_{1}^{2}+s_{2}^{2}\right)^{1 / 2} \leq-\sqrt{2} K V^{1 / 2} .
\end{aligned}
$$

In our computer simulations we assumed the parameter values as in MRC, and we also set $\tilde{k}_{s}=0.0, \tilde{d}_{1}=0.0, \tilde{d}_{2}=0.0$. The values for the constants were chosen as $K=4, \gamma=2, \lambda=2, \alpha=3$ and $\beta=5$. Initial conditions are the same as in MRC case. Error trajectory and controller time-history 


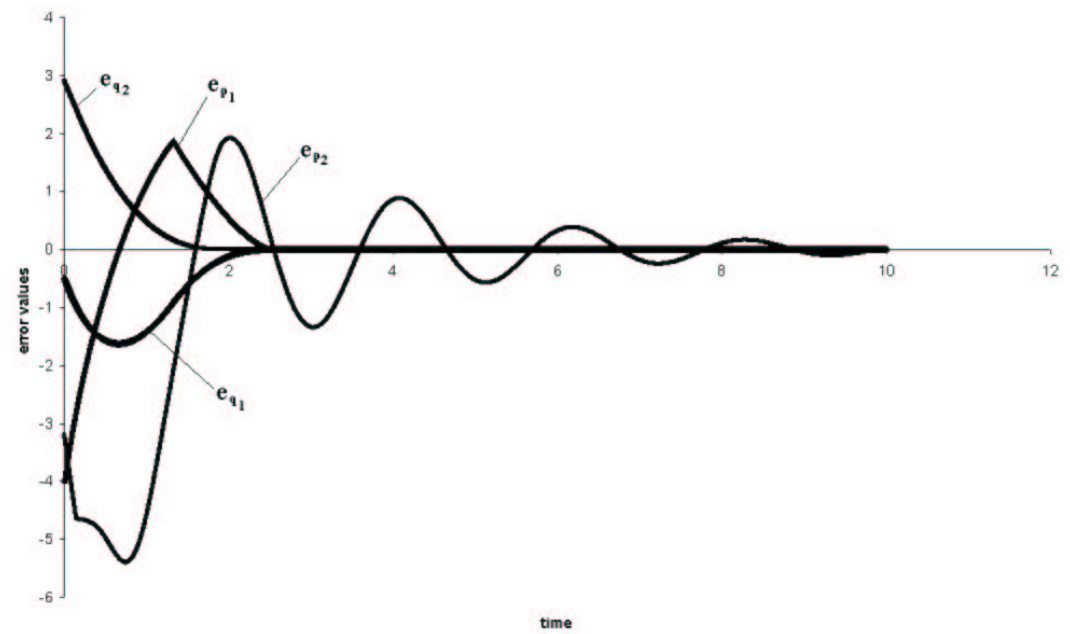

(a)

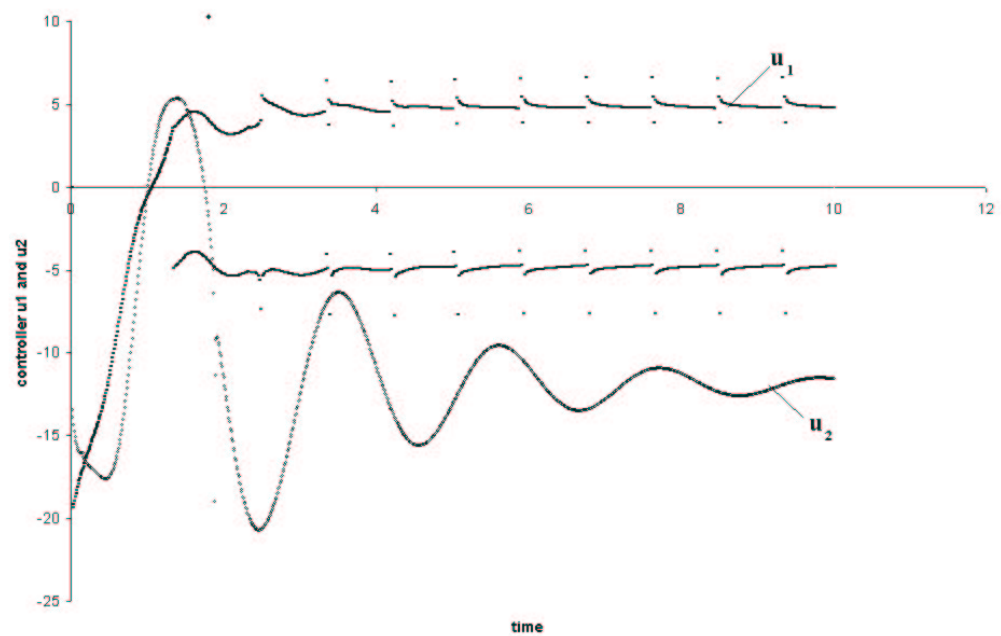

(b)

FiguRE 4: (a) Error convergence for MRAC (b) Controller for MRAC 
3 Model reference adaptive control - MRAC

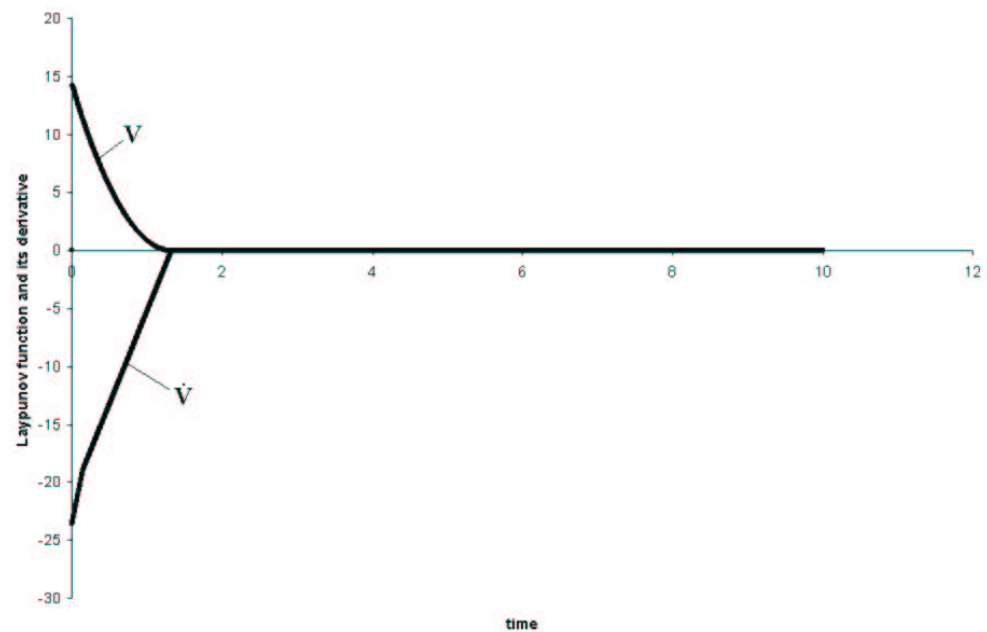

(a)

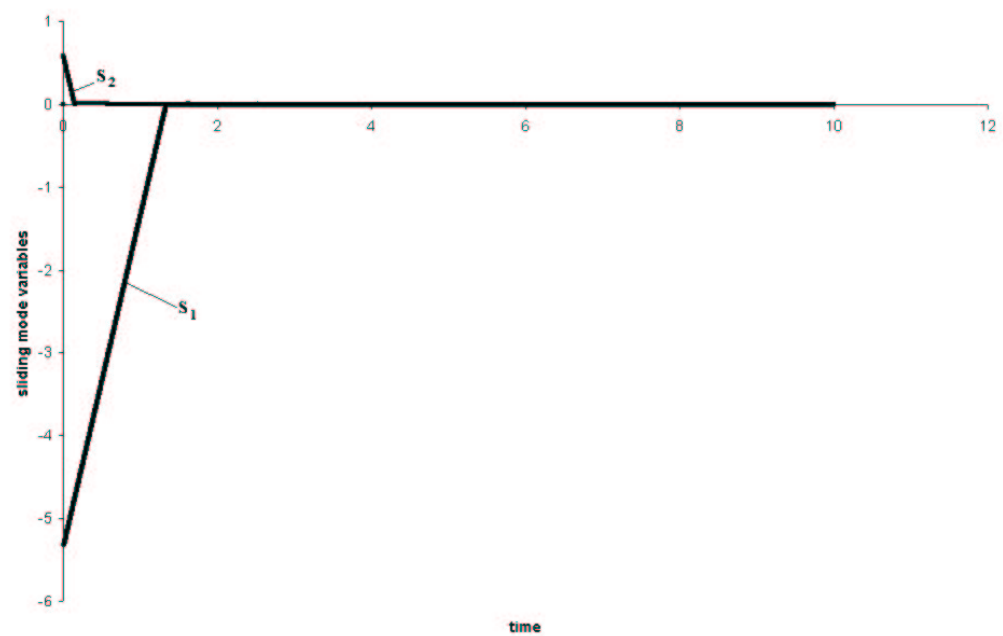

(b)

Figure 5: (a) Lyapunov function and its derivative (b) Sliding mode variables 
are shown on Figure 4. The time history of the Lyapunov and its derivative, together with the sliding mode variables are shown in Figure 5.

The convergence times to the $\epsilon$-envelope of the origin are $T_{c}=9.47$ for $\epsilon=0.1$ and $T_{c}=10.65$ for $\epsilon=0.05$. In some cases the convergence times are even shorter than in non-adaptive case despite uncertainty introduced to the system. It shows robustness of the adaptive scheme.

\section{$4 \quad$ Stability}

The sliding mode controller that we used up to now does not guarantee stability. We require a new control law that secures stability of the system.

\subsection{Supervisory controller}

Consider the two level control system with supervisory controller, illustrated on Figure 6.

The idea is to introduce a second-level controller designed to guarantee stability and take advantage of the properties of the sliding mode controller as the main controller without compromising its performance. The secondlevel controller acts as a supervisory controller, that is, when the sliding mode controller leads to instability of the system, it starts working to return stability of the system. Otherwise it remains idle.

We show here how develop the supervisory controller for MRC, as the controller for MRAC would be the same with adaptive terms added.

Denote our control law (10) as $u_{i}^{\mathrm{SM}}(\boldsymbol{x})=Q_{i}^{F}(\boldsymbol{x})$. Our task then is to design the second level controller that would guarantee that the control system 


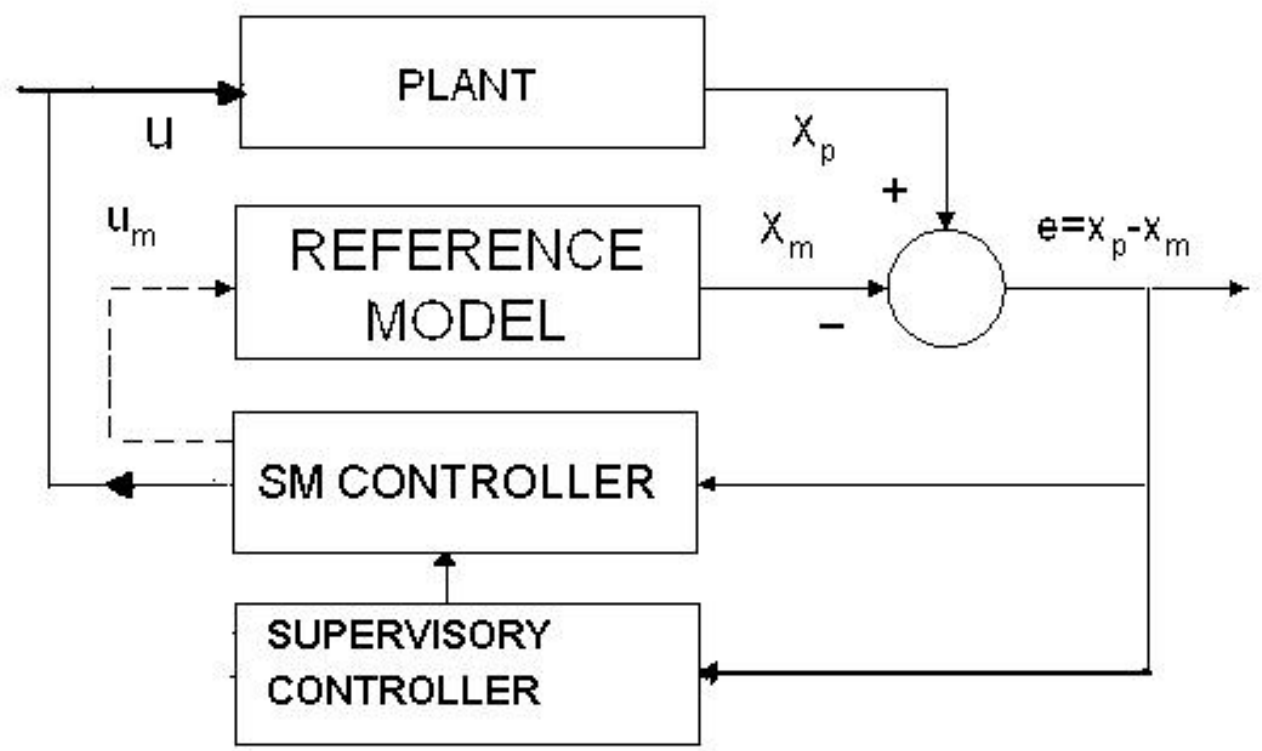

Figure 6: Two level control system. 
is globally stable, that is

$$
\|\boldsymbol{x}\| \leq B \quad \text { for every } \quad t>0,
$$

where $B>0$ is an arbitrary constant chosen by design. To this end we append the sliding mode controller $u_{i}^{\mathrm{SM}}, i=1, \ldots, n$, with a supervisory controller $u_{i}^{S}(\boldsymbol{x}), i=1, \ldots, n$, which is zero inside the ball $B_{x}=\{\boldsymbol{x}:\|\boldsymbol{x}\| \leq$ $B\}$ and is activated only when the system's trajectory reaches the boundary of $B_{x}$. Define the two-level controller as

$$
u_{i}=u_{i}^{\mathrm{SM}}(\boldsymbol{x})+I_{s}(\boldsymbol{x}) u_{i}^{S}(\boldsymbol{x}), \quad i=1, \ldots, n,
$$

where

$$
I_{s}(\boldsymbol{x})= \begin{cases}1, & \text { for }\|x\| \geq B \\ 0, & \text { otherwise }\end{cases}
$$

We design $u_{i}^{S}$ such that $\|\boldsymbol{x}\| \leq B$ for all $t>0$.

\subsection{Stable controller for sliding mode}

Assume that the system and model are defined by (1) and (2), and that the model Hamiltonian has the form

$$
\mathcal{H}_{m}\left(\boldsymbol{q}_{m}, \boldsymbol{p}_{m}\right)=\frac{1}{2} \sum_{i=1}^{n} a_{m_{i}} p_{m_{i}}^{2}+f_{m}\left(\boldsymbol{q}_{m}\right),
$$

where $a_{m_{i}}>0$ is a constant, $f_{m}(\cdot)$ is a known function, equivalent to a potential energy function. For the plant Hamiltonian $\mathcal{H}$, the state equation for

$$
\dot{q}_{i}=p_{i} / C_{i}(\boldsymbol{q}),
$$

where functions $C_{i}(\boldsymbol{q}) \neq 0$ and such that there exist estimation functions $f_{\text {est }_{i}}\left(\boldsymbol{e}_{q}, \boldsymbol{q}\right)$ satisfying

$$
\frac{\partial \mathcal{H}_{m}(\boldsymbol{e})}{\partial e_{q_{i}}}\left(\frac{p_{i}}{C_{i}(\boldsymbol{q})}-a_{m_{i}} p_{m_{i}}\right) \leq f_{\mathrm{est}_{i}} e_{p_{i}} .
$$


Furthermore, the plant's momenta are in the following form:

$$
\dot{p}_{i}=-f_{p_{i}}(\boldsymbol{p}, \boldsymbol{q}, \boldsymbol{a})+Q_{i}^{D}+Q_{i}^{F},
$$

where $f_{p_{i}}(\cdot)$ is a known nonlinear function polluted by uncertainty.

Then the plant dynamics for $i=1, \ldots, n$ are

$$
\begin{aligned}
\dot{q}_{i} & =\frac{p_{i}}{C_{i}(\boldsymbol{q})}, \\
\dot{p}_{i} & =-\frac{\partial \mathcal{H}_{p}(\boldsymbol{q}, \boldsymbol{p})}{\partial q_{i}}+Q_{i}^{D}(\boldsymbol{q}, \boldsymbol{p})+Q_{i}^{F}(\boldsymbol{q}, \boldsymbol{p}, \boldsymbol{u}) .
\end{aligned}
$$

The reference model for $i=1, \ldots, n$ is

$$
\begin{aligned}
& \dot{q}_{m_{i}}=a_{m_{i}} p_{m_{i}}, \\
& \dot{p}_{m_{i}}=-\frac{\partial f_{m}\left(\boldsymbol{q}_{m}\right)}{\partial q_{m_{i}}}+Q_{m_{i}}^{D}\left(\boldsymbol{q}_{m}, \boldsymbol{p}_{m}, \boldsymbol{d}_{m}\right)+Q_{m_{i}}^{F}\left(\boldsymbol{q}_{m}, \boldsymbol{p}_{m}, \boldsymbol{u}_{m}\right) .
\end{aligned}
$$

Equations (43) and (44) give the following error dynamics:

$$
\begin{aligned}
\dot{\boldsymbol{e}}_{q_{i}}= & \frac{p_{i}}{C_{i}(\boldsymbol{q})}-a_{m_{i}} p_{m_{i}} \\
\dot{\boldsymbol{e}}_{p_{i}}= & -\frac{\partial \mathcal{H}_{p}(\boldsymbol{q}, \boldsymbol{p})}{\partial q_{i}}+\frac{\partial f_{m}\left(\boldsymbol{q}_{m}\right)}{\partial q_{m_{i}}}+Q_{i}^{D}(\boldsymbol{q}, \boldsymbol{p}) \\
& -Q_{m_{i}}^{D}\left(\boldsymbol{q}_{m}, \boldsymbol{p}_{m}\right)+Q_{i}^{F}(\boldsymbol{q}, \boldsymbol{p}, \boldsymbol{u}) \\
& -Q_{m_{i}}^{F}\left(\boldsymbol{q}_{m}, \boldsymbol{p}_{m}, \boldsymbol{u}_{m}\right), \quad i=1, \ldots, n .
\end{aligned}
$$

Consider explicitly the terms of the controller $u_{i}$, see (10):

$$
\begin{aligned}
u_{i}= & \frac{\partial H_{p}}{\partial q_{i}}-Q_{i}^{D}(\boldsymbol{q}, \boldsymbol{p}, \boldsymbol{d}) \\
& -\frac{1}{f_{i}^{1}}\left(f_{i}^{2}(\boldsymbol{q}, \boldsymbol{p})-\dot{E}_{q_{m_{i}}}+\frac{\alpha}{\beta} \lambda e_{q_{i}}^{\alpha / \beta-1}\left(E_{q_{i}}-E_{q_{m_{i}}}\right)\right) \\
& -\frac{K}{f_{i}^{1}} \operatorname{sgn}\left(s_{i}\right)+I_{s} u_{i}^{S}, \quad i=1, \ldots, n,
\end{aligned}
$$


where in this particular case we write terms $\dot{E}_{q_{m_{i}}}$ and $E_{q_{i}}-E_{q_{m_{i}}}$ as

$$
\begin{aligned}
\dot{E}_{q_{m_{i}}} & =a_{m_{i}} \dot{p}_{m_{i}}=a_{m_{i}}\left(-\frac{\partial f_{m}}{\partial q_{m_{i}}}+Q_{m_{i}}^{D}+Q_{m_{i}}^{F}\right), \\
E_{q_{i}}-E_{q_{m_{i}}} & =\frac{p_{i}}{C_{i}(\boldsymbol{q})}-a_{m_{i}} p_{m_{i}}, \quad i=1, \ldots, n,
\end{aligned}
$$

Then substituting (47) and (48) into (46) we obtain for $i=1, \ldots, n$

$$
\begin{aligned}
u_{i}= & \frac{\partial H_{p}}{\partial q_{i}}-Q_{i}^{D}-\frac{1}{f_{i}^{1}}\left(f_{i}^{2}+a_{m_{i}} \frac{\partial f_{m}}{\partial q_{m_{i}}}-a_{m_{i}} Q_{m_{i}}^{D}-a_{m_{i}} Q_{m_{i}}^{F}\right. \\
& \left.+\frac{\alpha}{\beta} \lambda e_{q_{i}}^{\alpha / \beta-1}\left(\frac{p_{i}}{C_{i}(\boldsymbol{q})}-a_{m_{i}} p_{m_{i}}\right)\right)-\frac{K}{f_{i}^{1}} \operatorname{sgn}\left(s_{i}\right)+I_{s} u_{i}^{S} \\
= & \frac{\partial H_{p}}{\partial q_{i}}-Q_{i}^{D}-\frac{f_{i}^{2}}{f_{i}^{1}}-\frac{a_{m_{i}}}{f_{i}^{1}} \frac{\partial f_{m}}{\partial q_{m_{i}}}+\frac{a_{m_{i}}}{f_{i}^{1}} Q_{m_{i}}^{D}+\frac{a_{m_{i}}}{f_{i}^{1}} Q_{m_{i}}^{F} \\
& -\frac{\alpha}{\beta} \lambda e_{q_{i}}^{\alpha / \beta-1}\left(\frac{p_{i}}{C_{i}(\boldsymbol{q})}-a_{m_{i}} p_{m_{i}}\right)-\frac{K}{f_{i}^{1}} \operatorname{sgn}\left(s_{i}\right)+I_{s} u_{i}^{S} .
\end{aligned}
$$

Replacing $Q_{i}^{F}$ with $u_{i}$ in (45) we obtain

$$
\begin{aligned}
\dot{\boldsymbol{e}}_{q_{i}}= & \frac{p_{i}}{C_{i}(\boldsymbol{q})}-a_{m_{i}} p_{m_{i}}, \\
\dot{\boldsymbol{e}}_{p_{i}}= & -\frac{f_{i}^{2}}{f_{i}^{1}}+\left(1-\frac{a_{m_{i}}}{f_{i}^{1}}\right)\left(\frac{\partial f_{m}}{\partial q_{m_{i}}}-Q_{m_{i}}^{D}-Q_{m_{i}}^{F}\right) \\
& -\frac{\alpha}{\beta} \lambda e_{q_{i}}^{\alpha / \beta-1}\left(\frac{p_{i}}{C_{i}(\boldsymbol{q})}-a_{m_{i}} p_{m_{i}}\right) \\
& -\frac{K}{f_{i}^{1}} \operatorname{sgn}\left(s_{i}\right)+I_{s} u_{i}^{S}, \quad i=1, \ldots, n .
\end{aligned}
$$

The above error dynamics constitutes the system to be stabilised. Using the Hamiltonian function for the model, we define the Lyapunov function

$$
V(\boldsymbol{e})=\mathcal{H}_{m}\left(\boldsymbol{e}_{q}, \boldsymbol{e}_{p}\right)=\frac{1}{2} \sum_{i=1}^{n} a_{m_{i}} e_{p_{i}}^{2}+f_{m}(\boldsymbol{e}) .
$$


We now evaluate the time derivative of $V$ :

$$
\begin{aligned}
\dot{V}= & \left(\frac{\partial \mathcal{H}_{m}}{\partial \boldsymbol{e}_{q}}\right)^{T} \dot{\boldsymbol{e}}_{q}+\left(\frac{\partial \mathcal{H}_{m}}{\partial \boldsymbol{e}_{p}}\right)^{T} \dot{\boldsymbol{e}}_{p} \\
= & \left(\frac{\partial \mathcal{H}_{m}}{\partial \boldsymbol{e}_{q}}\right)^{T}\left[\frac{\partial \mathcal{H}_{p}}{\partial \boldsymbol{p}}-\frac{\partial \mathcal{H}_{m}}{\partial \boldsymbol{p}_{m}}\right] \\
& +\left(\frac{\partial \mathcal{H}_{m}}{\partial \boldsymbol{e}_{p}}\right)^{T}\left[-\frac{\partial \mathcal{H}_{p}}{\partial \boldsymbol{q}}+\frac{\partial \mathcal{H}_{m}}{\partial \boldsymbol{q}_{m}}+\left(\boldsymbol{Q}^{D}-\boldsymbol{Q}_{m}^{D}\right)+\left(\boldsymbol{Q}^{F}-\boldsymbol{Q}_{m}^{F}\right)\right] .
\end{aligned}
$$

In the second term on the right-hand side add and subtract inside the bracket, the term $\boldsymbol{Q}_{m}^{D}(\boldsymbol{e})$, to obtain

$$
\begin{aligned}
\dot{V}(\boldsymbol{e})= & \left(\frac{\partial \mathcal{H}_{m}}{\partial \boldsymbol{e}_{p}}\right)^{T}\left(\boldsymbol{e}_{q}, \boldsymbol{e}_{p}\right) \boldsymbol{Q}_{m}^{D}(\boldsymbol{e})+\left(\frac{\partial \mathcal{H}_{m}}{\partial \boldsymbol{e}_{q}}\right)^{T}\left[\frac{\partial \mathcal{H}_{p}}{\partial \boldsymbol{p}}-\frac{\partial \mathcal{H}_{m}}{\partial \boldsymbol{p}_{m}}\right] \\
+ & \left(\frac{\partial \mathcal{H}_{m}}{\partial \boldsymbol{e}_{p}}\right)^{T}\left[-\frac{\partial \mathcal{H}_{p}}{\partial \boldsymbol{q}}+\frac{\partial \mathcal{H}_{m}}{\partial \boldsymbol{q}_{m}}+\left(\boldsymbol{Q}^{D}-\boldsymbol{Q}_{m}^{D}\right)\right. \\
& \left.+\left(\boldsymbol{Q}^{F}-\boldsymbol{Q}_{m}^{F}\right)-\boldsymbol{Q}_{m}^{D}(\boldsymbol{e})\right]
\end{aligned}
$$

Taking into account our assumptions about the special form of $\mathcal{H}_{m}$ gives

$$
\begin{aligned}
\dot{V}(\boldsymbol{e})= & \sum_{i=1}^{n} a_{m_{i}} e_{p_{i}} Q_{m_{i}}^{D}(\boldsymbol{e}) \\
& +\sum_{i=1}^{n} \frac{\partial f_{m}\left(\boldsymbol{e}_{q}\right)}{\partial e_{q_{i}}}\left(\frac{p_{i}}{C_{i}(\boldsymbol{q})}-a_{m_{i}} p_{m_{i}}\right) \\
& +\sum_{i=1}^{n} a_{m_{i}} e_{p_{i}}\left(-\frac{p_{i}}{C(\boldsymbol{q})}-a_{m_{i}} p_{m_{i}}\right) \\
& +\sum_{i=1}^{n} a_{m_{i}} e_{p_{i}}\left(-\frac{\partial \mathcal{H}_{p}}{\partial q_{i}}+\frac{\partial f_{m}}{\partial q_{m_{i}}} Q_{i}^{D}-Q_{m_{i}}^{D}-Q_{m_{i}}^{D}(\boldsymbol{e})\right. \\
& \left.+Q_{i}^{F}-Q_{m_{i}}^{F}\right)
\end{aligned}
$$




$$
\begin{aligned}
\leq \sum_{i=1}^{n} & a_{m_{i}} e_{p_{i}} Q_{m_{i}}^{D}(\boldsymbol{e})+\sum_{i=1}^{n} a_{m_{i}} e_{p_{i}}\left(f_{\mathrm{est}_{i}}-\frac{\partial \mathcal{H}_{p}}{\partial q_{i}}+\frac{\partial f_{m}}{\partial q_{m_{i}}}\right. \\
& \left.+Q_{i}^{D}-Q_{m_{i}}^{D}-Q_{m_{i}}^{D}(\boldsymbol{e})+Q_{i}^{F}-Q_{m_{i}}^{F}\right) .
\end{aligned}
$$

Replacing $Q_{i}^{F}$ with the control $u_{i}$, see (49), we obtain

$$
\begin{aligned}
& \dot{V}(\boldsymbol{e}) \leq \sum_{i=1}^{n} a_{m_{i}} e_{p_{i}} Q_{m_{i}}^{D}(\boldsymbol{e})+\sum_{i=1}^{n} a_{m_{i}} e_{p_{i}}\left[f_{\mathrm{est}_{i}}-\frac{f_{i}^{2}}{f_{i}^{1}}-Q_{m_{i}}^{D}(\boldsymbol{e})\right. \\
&+\left(1-\frac{a_{m_{i}}}{f_{i}^{1}}\right) \frac{\partial f_{m}}{\partial q_{m_{i}}}-\left(1-\frac{a_{m_{i}}}{f_{i}^{1}}\right) Q_{m_{i}}^{D} \\
&-\left(1-\frac{a_{m_{i}}}{f_{i}^{1}}\right) Q_{m_{i}}^{F}-\frac{\alpha}{\beta} \frac{\lambda}{f_{i}^{1}} e_{q_{i}}^{\alpha / \beta-1}\left(\frac{p_{i}}{C_{i}(\boldsymbol{q})}-a_{m_{i}} p_{m_{i}}\right) \\
&\left.-\frac{K}{f_{i}^{1}} \operatorname{sgn}\left(s_{i}\right)+I_{s} u_{i}^{S}\right] \\
&=\sum_{i=1}^{n} a_{m_{i}} e_{p_{i}} Q_{m_{i}}^{D}(\boldsymbol{e})+\sum_{i=1}^{n} a_{m_{i}} e_{p_{i}}\left[f_{\mathrm{est}_{i}}-\frac{f_{i}^{2}}{f_{i}^{1}}-Q_{m_{i}}^{D}(\boldsymbol{e})\right. \\
& \quad+\left(1-\frac{a_{m_{i}}}{f_{i}^{1}}\right)\left(\frac{\partial f_{m}}{\partial q_{m_{i}}}-Q_{m_{i}}^{D}-Q_{m_{i}}^{F}\right) \\
&-\frac{\alpha}{\beta} \frac{\lambda}{f_{i}^{1}} e_{q_{i}}^{\alpha / \beta-1}\left(\frac{p_{i}}{C_{i}(\boldsymbol{q})}-a_{m_{i}} p_{m_{i}}\right) \\
&\left.-\frac{K}{f_{i}^{1}} \operatorname{sgn}\left(s_{i}\right)+I_{s} u_{i}^{S}\right], \quad i=1, \ldots, n .
\end{aligned}
$$

Assume now that $\|\boldsymbol{e}\| \geq B$, that is $I_{s}=1$. Let our controller $u_{i}^{S}$ be designed as

$$
\begin{aligned}
& u_{i}^{S}= \frac{f_{i}^{2}}{f_{i}^{1}}-f_{\text {est }_{i}}+Q_{m_{i}}^{D}(\boldsymbol{e})-\left(1-\frac{a_{m_{i}}}{f_{i}^{1}}\right)\left(\frac{\partial f_{m}}{\partial q_{m_{i}}}-Q_{m_{i}}^{D}-Q_{m_{i}}^{F}\right) \\
&+\frac{\alpha}{\beta} \frac{\lambda}{f_{i}^{1}} e_{q_{i}}^{\alpha / \beta-1}\left(\frac{p_{i}}{C_{i}(\boldsymbol{q})}-a_{m_{i}} p_{m_{i}}\right)+\frac{K}{f_{i}^{1}} \operatorname{sgn}\left(s_{i}\right)-e_{p_{i}}, \\
& \quad i=1, \ldots, n .
\end{aligned}
$$


Note, that for $\|\boldsymbol{e}\| \geq B$ we can simplify the expression for $u_{i}$. Indeed, substituting (56) into (49) we obtain for $i=1, \ldots, n$

$$
u_{i}=\frac{\partial H_{p}}{\partial q_{i}}-\frac{f_{i}^{2}}{f_{i}^{1}}-f_{\mathrm{est}_{i}}-\frac{\partial f_{m}}{\partial q_{m_{i}}}-Q_{i}^{D}+Q_{m_{i}}^{D}+Q_{m_{i}}^{D}(\boldsymbol{e})+Q_{m_{i}}^{F}-e_{p_{i}} .
$$

Substituting (56) into (55) gives

$$
\dot{V}(\boldsymbol{e}) \leq \sum_{i=1}^{n} a_{m_{i}} e_{p_{i}} Q_{m_{i}}^{D}(\boldsymbol{e})-\sum_{i=1}^{n} a_{m_{i}} e_{p_{i}}^{2} \leq 0 .
$$

This shows that the controller $u_{i}^{S}, i=1, \ldots, n$, secures decreasing of $\|\boldsymbol{e}\|$ if $\|\boldsymbol{e}\| \geq B$. Consequently, if the initial condition lies within the ball $B_{x}$, that is, $\|\boldsymbol{e}\| \leq B$, then $\|\boldsymbol{e}\| \leq B$ for all $t>0$.

The discontinuous character of function $I_{s}(\cdot)$ may cause chattering in the region across boundary of $B_{x}$. To minimise such oscillations we may introduce a modified version of $I_{s}(\cdot)$ :

$$
I_{s}= \begin{cases}0, & \text { if }\|\boldsymbol{e}\|<\epsilon, \\ \frac{\|\boldsymbol{e}\|-\epsilon}{B-\epsilon}, & \text { if } \epsilon \leq\|\boldsymbol{e}\| \leq B \\ 1, & \text { if }\|\boldsymbol{e}\| \geq B\end{cases}
$$

where constant $0<\epsilon<B$ is given by design.

\subsection{Robot illustration}

We easily identify terms in (10), (49) and (56):

$$
\begin{aligned}
& f_{1}^{1}=1.0, \quad f_{1}^{2}=0 \\
& f_{2}^{1}=\frac{1}{C\left(q_{c}\right)}, \quad f_{2}^{2}=-\frac{\dot{C}\left(q_{c}\right)}{C^{2}\left(q_{c}\right)} p_{2}
\end{aligned}
$$


and

$$
\begin{aligned}
f_{\mathrm{est}_{1}} & =\frac{k_{s_{m}}}{m_{m_{a}}+m_{m_{\ell}}} e_{q_{1}}, \\
f_{\mathrm{est}_{2}} & = \begin{cases}s_{m} e_{q_{2}}\left(p_{2} / C\left(q_{c}\right)-p_{m_{2}}\right) / e_{p_{2}}, & \text { for }\left|e_{p_{2}}\right|>\epsilon, \\
s_{m} e_{q_{2}}, & \text { otherwise, }\end{cases} \\
E_{q_{m_{1}}} & =p_{m_{1}}, \quad E_{q_{m_{2}}}=p_{m_{2}}, \\
\dot{E}_{q_{m_{1}}} & =E_{p_{m_{1}}}=-\frac{k_{s_{m}}}{m_{m_{a}}+m_{m_{\ell}}} q_{m_{1}}+Q_{m_{1}}^{D}+Q_{m_{1}}^{F}, \\
\dot{E}_{q_{m_{2}}} & =E_{p_{m_{2}}}=-s_{m} q_{m_{2}}+Q_{m_{2}}^{D}+Q_{m_{2}}^{F}, \\
a_{m_{1}} & =a_{m_{2}}=1, \\
C_{1}\left(q_{c}\right) & \equiv 1, \quad C_{2}\left(q_{c}\right)=C\left(q_{c}\right), \\
f_{m} & =\frac{1}{2}\left(\frac{k_{s_{m}}}{m_{m_{a}}+m_{m_{\ell}}} q_{m_{1}}^{2}+s_{m} q_{m_{2}}^{2}\right) \\
\frac{\partial f_{m}}{\partial q_{m_{1}}} & =\frac{k_{s_{m}}}{m_{m_{a}}+m_{m_{\ell}}} q_{m_{1}}, \\
\frac{\partial f_{m}}{\partial q_{m_{2}}} & =s_{m} q_{m_{2}} .
\end{aligned}
$$

Note that

$$
\begin{aligned}
\frac{\partial H_{p}}{\partial q_{1}}=- & {\left[\left(\frac{3}{4} m_{a}+m_{\ell}\right) \frac{q_{1}}{\left(m_{a}+m_{\ell}\right)}\right.} \\
& \left.+\left(\frac{1}{8} m_{a}+\frac{2}{3} m_{\ell}\right) \frac{\ell}{\sqrt{m_{a}+m_{\ell}}}\right]\left(\frac{p_{2}}{C\left(q_{c}\right)}\right)^{2} \\
& +\frac{k_{s}}{\left(m_{a}+m_{\ell}\right)} q_{1}, \\
\frac{\partial H_{p}}{\partial q_{2}}= & 0 .
\end{aligned}
$$


Then we write the control law according to (46). Observe that $u_{1}^{\mathrm{SM}}$ and $u_{2}^{\mathrm{SM}}$ are (20) and (21):

$$
\begin{aligned}
u_{1}^{\mathrm{SM}}=- & {\left[\left(\frac{3}{4} m_{a}+m_{\ell}\right) \frac{q_{1}}{\left(m_{a}+m_{\ell}\right)}\right.} \\
& \left.+\left(\frac{1}{8} m_{a}+\frac{2}{3} m_{\ell}\right) \frac{\ell}{\sqrt{m_{a}+m_{\ell}}}\right]\left(\frac{p_{2}}{C\left(q_{c}\right)}\right)^{2} \\
+ & \frac{k_{s}}{\left(m_{a}+m_{\ell}\right)} q_{1}-Q_{1}^{D}+Q_{m_{1}}^{D}+Q_{m_{1}}^{F} \\
& -\frac{k_{s_{m}}}{\left(m_{m_{a}}+m_{m_{\ell}}\right)} q_{m_{1}}-\frac{\alpha}{\beta} \lambda e_{q_{1}}^{\alpha / \beta-1} e_{p_{1}}-K \operatorname{sgn}\left(s_{1}\right), \\
u_{2}^{\mathrm{SM}}= & -Q_{2}^{D}+\frac{\dot{C}\left(q_{c}\right) p_{2}}{C\left(q_{c}\right)}-C\left(q_{c}\right) s_{m} q_{m_{2}}+C\left(q_{c}\right) Q_{m_{2}}^{D}+C\left(q_{c}\right) Q_{m_{2}}^{F} \\
& -C\left(q_{c}\right) \frac{\alpha}{\beta} \lambda e_{q_{2}}^{\alpha / \beta-1}\left(\frac{p_{2}}{C\left(q_{c}\right)}-p_{m_{2}}\right)-C\left(q_{c}\right) K \operatorname{sgn}\left(s_{2}\right),
\end{aligned}
$$

and our supervisory controller is

$$
\begin{aligned}
u_{1}^{S}= & -\frac{k_{s_{m}}}{\left(m_{m_{a}}+m_{m_{\ell}}\right)} e_{q_{1}}+Q_{m_{1}}^{D}(\boldsymbol{e})+\frac{\alpha}{\beta} \frac{\lambda}{f_{1}^{1}} e_{q_{1}}^{\alpha / \beta-1} e_{p_{1}} \\
& +K \operatorname{sgn}\left(s_{1}\right)-e_{p_{1}} \\
u_{2}^{S}= & -\frac{\dot{C}\left(q_{c}\right) p_{2}}{C\left(q_{c}\right)}-f_{\mathrm{est}_{2}}+Q_{m_{2}}^{D}(\boldsymbol{e}) \\
& -\left(1-C\left(q_{c}\right)\right)\left(s_{m} q_{m_{2}}-Q_{m_{2}}^{D}-Q_{m_{2}}^{F}\right) \\
& +C\left(q_{c}\right) \frac{\alpha}{\beta} \lambda e_{q_{2}}^{\alpha / \beta-1}\left(\frac{p_{2}}{C\left(q_{c}\right)}-p_{m_{2}}\right) \\
& +C\left(q_{c}\right) K \operatorname{sgn}\left(s_{2}\right)-e_{p_{2}} .
\end{aligned}
$$

Then the control law for $\|\boldsymbol{e}\| \geq B$, that is $I_{s}=1$, is

$$
u_{1}=u_{1}^{\mathrm{SM}}+u_{1}^{S}
$$




$$
\begin{aligned}
= & -Q_{0}\left(\frac{p_{2}}{C\left(q_{c}\right)}\right)^{2}+\left(\frac{k_{s}}{\left(m_{a}+m_{\ell}\right)}-\frac{k_{s_{m}}}{\left(m_{m_{a}}+m_{m_{\ell}}\right)}\right) q_{1} \\
& -Q_{1}^{D}+Q_{m_{1}}^{D}+Q_{m_{1}}^{F}+Q_{m_{1}}^{D}(\boldsymbol{e})-e_{p_{1}}, \\
u_{2}= & u_{2}^{\mathrm{SM}}+u_{2}^{S} \\
= & -f_{\mathrm{est}_{2}}-s_{m} q_{m_{2}}-Q_{2}^{D}+Q_{m_{2}}^{D}+Q_{m_{2}}^{F}+Q_{m_{2}}^{D}(\boldsymbol{e})-e_{p_{2}} .
\end{aligned}
$$

Now we evaluate the time derivative of $V$ :

$$
\begin{aligned}
\dot{V}= & \frac{k_{s_{m}}}{\left(m_{m_{a}}+m_{m_{\ell}}\right)} e_{q_{1}} e_{p_{1}}+s_{m} e_{q_{2}}\left(\frac{1}{C\left(q_{c}\right)} p_{2}-p_{m_{2}}\right) \\
+ & {\left[Q_{0}\left(\frac{p_{2}}{C\left(q_{c}\right)}\right)^{2}-\frac{k_{s}}{m_{a}+m_{l}} q_{1}+\frac{k_{s_{m}}}{m_{m_{a}}+m_{m_{\ell}}} q_{m_{1}}\right.} \\
& \left.+Q_{1}^{D}-Q_{m_{1}}^{D}+Q_{1}^{F}-Q_{m_{1}}^{F}\right] e_{p_{1}} \\
& +\left(s_{m} q_{m_{2}}+Q_{2}^{D}-Q_{m_{2}}^{D}+Q_{2}^{F}-Q_{m_{2}}^{F}\right) e_{p_{2}} .
\end{aligned}
$$

We add and subtract inside the $e_{p_{i}}$-brackets the term $Q_{m_{i}}^{D}(\boldsymbol{e})=-d_{m_{i}} e_{p_{i}}$ :

$$
\begin{aligned}
\dot{V}= & -\sum_{i=1}^{2} d_{m_{i}} e_{p_{i}}^{2}+\frac{k_{s_{m}}}{\left(m_{m_{a}}+m_{m_{\ell}}\right)} e_{q_{1}} e_{p_{1}}+s_{m} e_{q_{2}}\left(\frac{1}{C\left(q_{c}\right)} p_{2}-p_{m_{2}}\right) \\
+ & e_{p_{1}}\left[Q_{0}\left(\frac{p_{2}}{C\left(q_{c}\right)}\right)^{2}-\frac{k_{s}}{m_{a}+m_{l}} q_{1}+\frac{k_{s_{m}}}{m_{m_{a}}+m_{m_{\ell}}} q_{m_{1}}\right. \\
& \left.+Q_{1}^{D}-Q_{m_{1}}^{D}-Q_{m_{1}}^{D}(\boldsymbol{e})+Q_{1}^{F}-Q_{m_{1}}^{F}\right] \\
+ & e_{p_{2}}\left(s_{m} q_{m_{2}}+Q_{2}^{D}-Q_{m_{2}}^{D}-Q_{m_{2}}^{D}(\boldsymbol{e})+Q_{2}^{F}-Q_{m_{2}}^{F}\right) \\
\leq & -\sum_{i=1}^{2} d_{m_{i}} e_{p_{i}}^{2} \\
& +e_{p_{1}}\left[Q_{0}\left(\frac{p_{2}}{C\left(q_{c}\right)}\right)^{2}+\left(\frac{k_{s_{m}}}{m_{m_{a}}+m_{m_{\ell}}}-\frac{k_{s}}{m_{a}+m_{l}}\right) q_{1}\right. \\
& \left.+Q_{1}^{D}-Q_{m_{1}}^{D}-Q_{m_{1}}^{D}(\boldsymbol{e})+Q_{1}^{F}-Q_{m_{1}}^{F}\right]
\end{aligned}
$$




$$
\begin{aligned}
& +e_{p_{2}}\left[f_{\text {est }_{2}}+s_{m} q_{m_{2}}+Q_{2}^{D}-Q_{m_{2}}^{D}\right. \\
& \left.\quad-Q_{m_{2}}^{D}(\boldsymbol{e})+Q_{2}^{F}-Q_{m_{2}}^{F}\right] .
\end{aligned}
$$

After replacing $Q_{1}^{F}$ and $Q_{2}^{F}$ with $u_{1}$ and $u_{2}$ in (68) we obtain

$$
\dot{V}=-\sum_{i=1}^{2} d_{m_{i}} e_{p_{i}}^{2}+\sum_{i=1}^{2} e_{p_{i}}^{2} \leq 0 .
$$

This proves stability of the origin.

\subsection{Computer simulation with supervisory controller}

We ran the simulation for our previous example with the same initial data and the radius was chosen as $B=4.2$. The values for the sliding mode constants were chosen as $K=4, \gamma=2, \lambda=2, \alpha=3$ and $\beta=5$. The diameter of the ball $B_{x}$ is chosen as $B=4.0$. As the norm in the error-space we have chosen $\|\boldsymbol{e}\|=\max \left\{\left|e_{q_{1}}\right|,\left|e_{q_{2}}\right|,\left|e_{p_{1}}\right|,\left|e_{p_{2}}\right|\right\}$. This norm gives us a better insight than the Euclidean norm as we are usually interested in keeping every single trajectory, that is every component of the vector $\boldsymbol{e}$, below a certain value (in our case $B$ ) whereas the Euclidean norm gives us a length of $\boldsymbol{e}$ in four-dimensional space. The results from one of many simulations are shown on Figure 7. See the supervisory controller acts only during brief period of time to secure stability of the system.

The impact of the supervisory controller is most visible on the $e_{p_{2}}$-trajectory. In the lower left side of the diagram there is characteristic chattering visible for a short period of time when supervisory controller switches on and off.

\subsection{Asymptotic stability versus sliding mode}

We ran two series (Series I and II) of computer simulations to compare the performance of the controller implementing an asymptotic stability (AS) tech- 
(a)
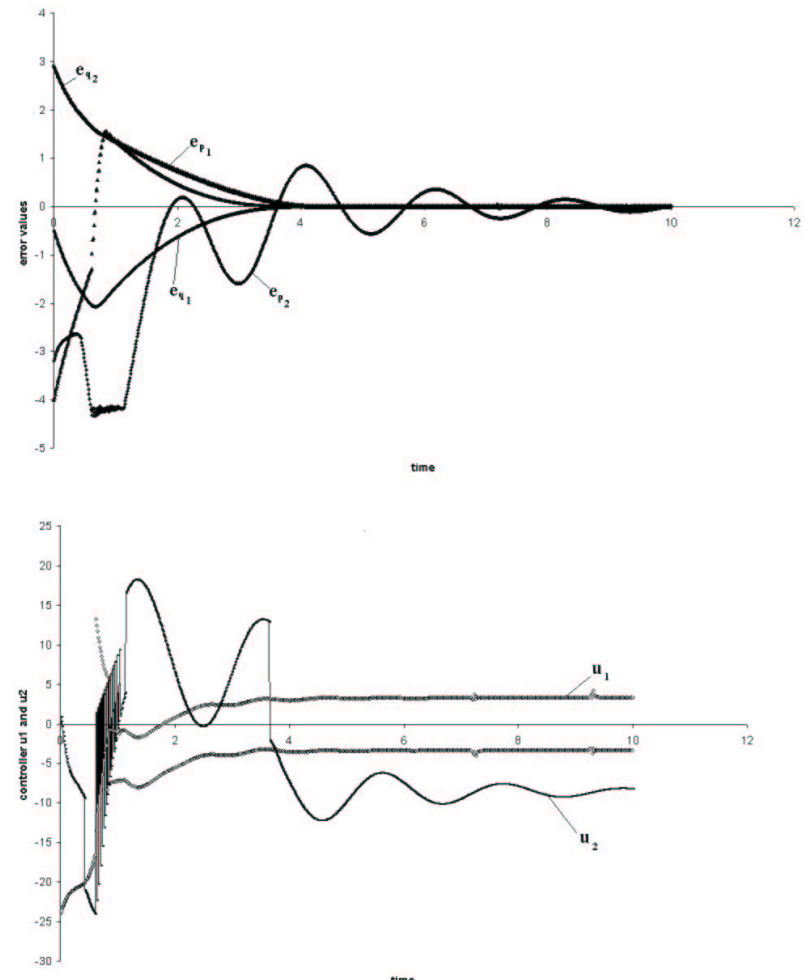

(b)

(c)

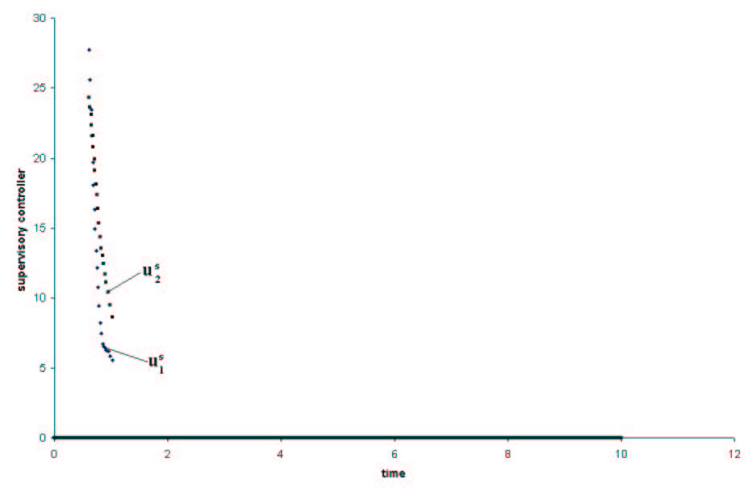

Figure 7: (a) Supervisory controller - error trajectories (b) Controller (c) Supervisory controller 
nique, developed in [3], and the sliding mode (SM) controller. Detailed discussion including simulations results and diagrams can be found in [5]. Two major factors were taken into account in this comparison: convergence time, and magnitude of the control force. Mathematical formalism secures finite time convergence for the SM-control which gives it apparently an advantage over the AS control method, but the results obtained did not support the SM controller claim. Both controllers achieve finite time convergence measured by $T_{c}$, the time error-trajectories entered an $\epsilon$-envelope of zero, where $\epsilon>0$ is an arbitrarily small number. For computational reasons this cannot be too small. In simulations we used $\epsilon=0.1$ (Series I and II) and $\epsilon=0.05$ (Series II). In both cases application of AS controller resulted in shorter $T_{c}$ times than SM-controller could produce. The explanation for the difference lay with the way the SM-controller handled the rotary link dynamics. Even though all but one error-trajectory converge very quickly to the $\epsilon$-envelope of zero, the trajectory of error in rotary joint momentum oscillated for an extended period of time, thus ruining the overall performance of the SM controller. In the case of the AS-controller, the same trajectory also oscillated but the amplitude of those oscillations was much lower and was quickly smoothed out.

We calculated also times $T_{f}$ at which the sliding mode variables reached terminal sliding mode $\left(s_{i}=0, i=1,2\right)$. These times are short, ranging from $T_{f}=0.31$ to $T_{f}=2.45$ (for $\epsilon=0.1$, Series I and II), and $T_{f}=0.43$ to $T_{f}=1.37$ (for $\epsilon=0.05$, Series II). However, the short time $T_{f}$ does not automatically translate into short times of error-trajectories convergence. There is no simple linear dependence between $T_{f}$ and $T_{c}$. In other words, the rate of reaching the SM surface does not correspond to the rate of errortrajectories convergence as defined above.

In the SM method we can manipulate constants $K_{1}$ and $\lambda$ to achieve the faster rate of convergence of sliding mode vector $s$ to zero. High values of $K_{1}$ and $\lambda$ may cause the time of error-trajectories convergence $T_{c}$ to become slightly longer. These high values can also cause error-trajectory bounce 
from its $\epsilon$-envelope of zero, thus increasing chattering.

In terms of energy expenditure by control force, the SM controller seems to have decisive advantage over the AS-controller; however, not in all cases.

Note that whenever there was a big misalignment between initial conditions of the plant and the reference model, the AS-controller required in the initial stage large control force to achieve tracking, whereas the SM-controller seemed much more 'frugal' in comparison, providing much better efficiency in terms of control force expenditure (especially in case when the motion of the joints is in opposite direction then that of the reference model). However, we noted in each series of simulations two exceptions that show that not for all initial conditions the SM-controller is more efficient.

The Asymptotic Stability controllers do their job effectively after initial settling down. The large values of control force in the initial phase are partly due to the imperfection of the estimation function $f_{\text {est }}$ used in our method [3]. However, the AS controllers generally exhibit a tendency to a substantial power consumption in the initial stage of tracking process. On the positive side, they are remarkably stable, quite predictable and quickly settle into a regular pattern.

To achieve a better accuracy in SM error-trajectories convergence and eliminate some of the 'bouncing' we would need to use finer time-increment in Runge-Kutta procedure for integration of the joint system of differential equations describing the system-model dynamics. The time-increment of $d t=0.001$ produces smoother error-trajectories but is unrealistic for practical applications. That was the reason we used as the finest increment $d t=0.01$. Application of boundary layer control would eliminate to some extent the chattering, but in all control systems with non-zero sampling interval the ideal convergence is unattainable [6]. 


\section{Conclusions}

We have shown here that the traditional sliding mode approach to MRC and MRAC works successfully in a rather general format for plant and reference models described in Hamiltonian format.

The development of adaptive control algorithms required linear parametrisation of the system's Hamiltonian. This linearisation is concerned mainly with parameters describing the system's mass properties.

A stabilising controller was successfully designed for the sliding mode method to ensure the stability of the resulting dynamical system. Our analysis for this stabilising supervisory controller was found to be sensitive to the choice of various estimation functions. Usually they are designed as discontinuous functions and this leads to switching control (especially when error-vector trajectories converge near zero). This in turn introduces chattering and in some cases, causes the control system to lose its ability to track the model trajectories. Such situations may occur when one or more error-vector components converge to zero.

A short discussion was given comparing the sliding mode control technique presented in this paper with the traditional asymptotic Lyapunov method previously published.

The sliding mode technique can be successfully implemented for systems in Hamiltonian form. The Hamiltonian form gives a physically meaningful formulation of the dynamics of control systems. Our use of the Hamiltonian of the reference model to obtain stability of the resulting system under sliding mode control, shows the use of how energy in this reference model plays a significant role in the control of the plant dynamics. 


\section{References}

[1] A. J. Koivo, Fundamentals for control of robotic manipulators (John Wiley and Sons, New York, 1989). E8

[2] J. M. Skowronski, Control of Nonlinear Mechanical Systems (Plenum Press, 1991). E3, E4

[3] R. J. Stonier and J. Zajaczkowski, Model Reference Control using Hamiltonian Functions, Proc. 4th International Conference on Control, Robotics and Vision ICARV'96 3 (1996) 2045-2049. E3, E37, E38

[4] R. J. Stonier and X. H. Yu, Finite Time Switching Control For Collision-Avoidance of Mobile Robots, Proc. 4th International Conference on Control, Automation, Robotics and Computer Vision ICARV'96 (1996) 1140-1144. E18

[5] J. Zajaczkowski, Model Reference Adaptive Control in Hamiltonian Systems, MSc Thesis, CQU, 2000. E37

[6] Man Zhihong, A. P. Paplinski, H. R. Wu, A robust MIMO terminal Sliding Mode Control Scheme for Rigid Robotic Manipulators IEEE Transactions on Automatic Control 39 (1994) 2464-2469. E38 